\title{
KOSZUL GORENSTEIN ALGEBRAS FROM COHEN-MACAULAY SIMPLICIAL COMPLEXES
}

\author{
ALESSIO D'ALÌ AND LORENZO VENTURELLO
}

\begin{abstract}
We associate with every pure flag simplicial complex $\Delta$ a standard graded Gorenstein $\mathbb{F}$-algebra $R_{\Delta}$ whose homological features are largely dictated by the combinatorics and topology of $\Delta$. As our main result, we prove that the residue field $\mathbb{F}$ has a $k$-step linear $R_{\Delta}$-resolution if and only if $\Delta$ satisfies Serre's condition $\left(S_{k}\right)$ over $\mathbb{F}$, and that $R_{\Delta}$ is Koszul if and only if $\Delta$ is Cohen-Macaulay over $\mathbb{F}$. Moreover, we show that $R_{\Delta}$ has a quadratic Gröbner basis if and only if $\Delta$ is shellable. We give two applications: first, we construct quadratic Gorenstein $\mathbb{F}$-algebras which are Koszul if and only if the characteristic of $\mathbb{F}$ is not in any prescribed set of primes. Finally, we prove that whenever $R_{\Delta}$ is Koszul the coefficients of its $\gamma$-vector alternate in sign, settling in the negative an algebraic generalization of a conjecture by Charney and Davis.
\end{abstract}

\section{Contents}

1. Introduction 1

2. Preliminaries 4

3. Bier balls and spheres $\quad 10$

4. From Bier balls to Gorenstein algebras: the ring $R_{\Delta} \quad 11$

5. When is $R_{\Delta}$ Koszul? 14

6. Quadratic Gröbner bases and shelling orders 22

7. The $\gamma$-vector of $R_{\Delta} \quad 27$

8. An Artinian reduction of $R_{\Delta}$ and a connection to work of Gondim and Zappalà 32

\section{INTRODUCTION}

It is a common line of thought in combinatorial commutative algebra to build correspondences between rings and objects of a more combinatorial nature. This can be insightful for both sides of the story. A prototypical example is Stanley-Reisner theory, where topological and combinatorial properties of simplicial complexes are studied via quotients of polynomial rings by squarefree monomial ideals. In this article we follow the same general principle and associate with any pure simplicial complex $\Delta$ a non-monomial Gorenstein standard graded $\mathbb{F}$-algebra $R_{\Delta}$, where $\mathbb{F}$ is a field. If the complex $\Delta$ is flag and satisfies some mild homological condition, the algebra $R_{\Delta}$ will moreover be quadratic.

2020 Mathematics Subject Classification. Primary: 05E40; Secondary: 16S37, 13H10, 13D02, 13 F65.

Key words and phrases. Koszul algebra, quadratic algebra, Gorenstein ring, flag simplicial complex, Cohen-Macaulay simplicial complex, minimal free resolution, Bier sphere, Nagata idealization. 
In combinatorics, Gorenstein algebras show up as Stanley-Reisner rings of homology spheres, as well as Ehrhart rings of certain lattice polytopes. While Gorenstein rings are very rich in structure and well-studied both in algebra and combinatorics, it is not always so easy to construct examples with prescribed features, e.g. with fixed $h$-vector. A classical and useful tool for this purpose is Nagata idealization, prominently featured in a recent article by Mastroeni, Schenck and Stillman [MSS21a].

A purely combinatorial way to construct a sphere from any simplicial complex $\Delta$ was introduced by Thomas Bier [Bie92] (see also [BPSZ05, Mur11, DFN19]): such a Bier sphere is PL and simultaneously contains both $\Delta$ and its Alexander dual. In this paper we will take a twist on this construction and crucially exploit a beautiful result by Terai and Yanagawa [Yan00, Corollary 3.7] relating Serre's $\left(S_{k}\right)$ property on $\Delta$ to the linearity of the resolution of the Stanley-Reisner ideal of the Alexander dual of $\Delta$.

Our recipe to build the ring $R_{\Delta}$ is as follows: said $I_{\Delta} \subseteq \mathbb{F}\left[x_{1}, \ldots, x_{n}\right]$ the Stanley-Reisner ideal of a pure simplicial complex $\Delta$ on $n$ vertices, we consider a new complex $\Gamma$ on $2 n$ vertices whose Stanley-Reisner ideal is

$$
I_{\Gamma}:=I_{\Delta}+\left(x_{i} y_{i}: i \in[n]\right) \subseteq \mathbb{F}\left[x_{1}, \ldots, x_{n}, y_{1}, \ldots, y_{n}\right] .
$$

Such a complex turns out to be a PL ball whose boundary is the Bier sphere associated with $\Delta$, as already noted by Murai in [Mur11]. Taking inspiration from [MSS21a], we then define the ring $R_{\Delta}$ as the Nagata idealization of the canonical module of $\mathbb{F}[\Gamma]$.

The main contribution of the present paper is to relate the homological features of the links of $\Delta$ with the behaviour of the minimal free resolution of the residue field $\mathbb{F}$ as an $R_{\Delta}$-module, under the extra assumption that $\Delta$ is flag (i.e., its Stanley-Reisner ideal is quadratically generated). In order to do this, we build some technical results comparing the resolutions of certain monomial ideals over different rings: see Corollary 5.11 and the lemmas preceding it. Our first result is as follows:

Theorem A (Theorem 5.1). Let $\Delta$ be a pure flag simplicial complex. The minimal resolution of the residue field $\mathbb{F}$ as an $R_{\Delta}$-module is linear for $k$ steps if and only if $\Delta$ satisfies Serre's condition $\left(S_{k}\right)$ over $\mathbb{F}$.

Note that flagness is indeed necessary for the resolution of $\mathbb{F}$ to have a chance to be linear: as the monomials generating $I_{\Delta}$ lie in a minimal generating set of the ideal defining $R_{\Delta}$, the second step of the $R_{\Delta}$-resolution of $\mathbb{F}$ fails to be linear whenever $\Delta$ is not flag. Serre's condition $\left(S_{k}\right)$ is an algebraic property that, when formulated for the Stanley-Reisner ring of $\Delta$, translates to a certain vanishing condition about the homology of its links. Reisner's criterion [Rei76] is precisely this translation when $k=d$, where $d=\operatorname{dim} \mathbb{F}[\Delta]=$ $\operatorname{dim}(\Delta)+1$ : when $\Delta$ satisfies $\left(S_{d}\right)$, we say that $\Delta$ is Cohen-Macaulay over $\mathbb{F}$.

The Cohen-Macaulayness of $\Delta$ over $\mathbb{F}$ translates to the $R_{\Delta}$-resolution of $\mathbb{F}$ being linear not just until the $d$-th step, but at every step. Standard graded algebras with this behaviour are called Koszul.

Theorem B (Corollary 5.3). Let $\Delta$ be a pure flag simplicial complex. The standard graded Gorenstein $\mathbb{F}$-algebra $R_{\Delta}$ is Koszul if and only if $\Delta$ is Cohen-Macaulay over $\mathbb{F}$.

We remark that this is not the first time the Koszul and Cohen-Macaulay properties have been brought together. Polo [Pol95] and Woodcock [Woo98] independently proved that 
the incidence algebra associated with a finite graded poset is a (noncommutative) Koszul algebra if and only if all the open intervals of the poset are Cohen-Macaulay, i.e. the order complex of every open interval is Cohen-Macaulay. In a similar vein, Peeva, Reiner and Sturmfels showed in [PRS98] that a graded pointed affine semigroup algebra is Koszul if and only if every open interval of the poset associated with the semigroup is Cohen-Macaulay. These results have later been unified by Reiner and Stamate in [RS10]. Another intriguing connection between Koszulness and Cohen-Macaulayness has been highlighted by Vallette in the context of operads [Val07]. Moreover, the study of the Koszul property for quadratic Gorenstein algebras has been the object of intensive research in the last few years [Mat18, MSS21a, MSS21b, MS20]. In particular, the results in [MSS21a, MSS21b, MS20] provide an almost fully complete characterization of the pairs $(c, r)$ for which there exists a non-Koszul quadratic Gorenstein algebra of codimension $c$ and Castelnuovo-Mumford regularity $r$ : the only cases that have not been settled yet are $(c, r)=(6,3)$ and $(c, r)=(7,3)$. Unfortunately, the techniques developed in this paper do not give further information on these cases, see Remark 5.14.

We find a pleasing application of Theorem $\mathrm{B}$ in Proposition 5.15. Fix a finite set $P$ of prime numbers. By considering certain simplicial manifolds $\Delta$ whose vanishing in homology depends on the characteristic of the field, we obtain standard graded quadratic Gorenstein $\mathbb{F}$-algebras $R_{\Delta}$ that are Koszul if and only if the characteristic of $\mathbb{F}$ is not in $P$.

Theorem B implies that checking Koszulness for the algebras $R_{\Delta}$ is equivalent to testing if $\Delta$ is Cohen-Macaulay. For instance, it suffices to compute the (finite) minimal free resolution of $\mathbb{F}[\Delta]$ to certify linearity of the (infinite) $R_{\Delta}$-resolution of $\mathbb{F}$. This is in stark contrast with the general behaviour of Koszul algebras. Indeed, working over a field $\mathbb{F}$ of characteristic zero, Roos [Roo93] constructed a family $\left(A_{\alpha}\right)_{\alpha \geq 2}$ of standard graded Artinian quadratic $\mathbb{F}$ algebras with fixed Hilbert series and such that the $\left(A_{\alpha}\right)$-resolution of $\mathbb{F}$ is linear for $\alpha$ steps, but fails to be so at the $(\alpha+1)$-st. Applying idealization to Roos' construction, McCullough and Seceleanu recently showed in [MS20, Theorem 5.1] that checking Koszulness is just as hard for quadratic Gorenstein algebras.

Since certifying Koszulness for a quadratic algebra is difficult, algebraists often resort to studying stronger conditions like the existence of a quadratic Gröbner basis for the defining ideal. Our next result shows that having a quadratic Gröbner basis for the algebras $R_{\Delta}$ corresponds indeed to a property of $\Delta$ which is much stronger than Cohen-Macaulayness.

Theorem $\mathbf{C}$ (Theorem 6.3). Let $\Delta$ be a pure flag simplicial complex. The Gorenstein $\mathbb{F}$ algebra $R_{\Delta}$ has a quadratic Gröbner basis if and only if $\Delta$ is a shellable complex.

As the ideal defining $R_{\Delta}$ has a characteristic-free generating set consisting of monomials and binomials of the form $\mathbf{m}-\mathbf{m}^{\prime}$ (Proposition 4.3), the existence of a quadratic Gröbner basis for such an object is a combinatorial property not depending on the choice of the field. The same is true for shellability of $\Delta$.

As a last topic, we investigate a numerical invariant related to the Hilbert series of $R_{\Delta}$. Gorenstein algebras are known to have a symmetric $h$-polynomial with nonnegative coefficients. Gal [Gal05] proposed the study of a certain linear transformation of these coefficients, called the $\gamma$-vector, and conjectured the nonnegativity of its entries in the case of StanleyReisner rings of flag $\mathbb{F}$-homology spheres. The validity of one of the inequalities given by Gal's 
conjecture is known as Charney-Davis conjecture [CD95] and has important implications in metric geometry: see [For07] for an excellent survey on the topic.

The Stanley-Reisner ring of a flag $\mathbb{F}$-homology sphere happens to be both Koszul and Gorenstein: using this observation as a starting point, Reiner and Welker began a more algebraic investigation of the Charney-Davis conjecture in [RW05]. An explicit generalization of the Charney-Davis conjecture in this direction can be found in a survey by Peeva and Stillman:

Question ([PS09, Problem 10.3]). Let $S / I$ be a Koszul Gorenstein algebra with $h$-vector $\left(h_{0}, h_{1}, \ldots, h_{2 e}\right)$. Is it true that $(-1)^{e}\left(h_{0}-h_{1}+h_{2}-\ldots+h_{2 e}\right) \geq 0$ ?

The answer turns out to be negative. This is a consequence of our last result:

Theorem D (Proposition 7.9). Let $\Delta$ be a pure $(d-1)$-dimensional simplicial complex. The vector $\gamma\left(R_{\Delta}\right)$ is given by $\gamma_{0}\left(R_{\Delta}\right)=1, \gamma_{1}\left(R_{\Delta}\right)=2 h_{1}(\Delta)+\sum_{k=2}^{d} h_{k}(\Delta)$, and

$$
\gamma_{i}\left(R_{\Delta}\right)=(-1)^{i-1} \sum_{k=2 i-1}^{d}\left(\left(\begin{array}{c}
k-i \\
i-1
\end{array}\right)+\left(\begin{array}{c}
k-i-1 \\
i-2
\end{array}\right)\right) h_{k}(\Delta)
$$

for $2 \leq i \leq\left\lfloor\frac{d+1}{2}\right\rfloor$. In particular, if $h(\Delta)$ has nonnegative entries, then $(-1)^{i-1} \gamma_{i}\left(R_{\Delta}\right) \geq 0$.

As the $h$-vector of a Cohen-Macaulay complex has nonnegative entries, Theorem D and Theorem $\mathrm{B}$ show that whenever the algebra $R_{\Delta}$ is Koszul, its $\gamma$-vector has entries which alternate in sign. In particular, when $\Delta$ is a $(d-1)$-dimensional Cohen-Macaulay flag complex with $d \equiv 3 \bmod 4$, the Charney-Davis quantity $\gamma_{\left\lfloor\frac{d+1}{2}\right\rfloor}\left(R_{\Delta}\right)$ is nonpositive. For an explicit example where $\gamma_{\left\lfloor\frac{d+1}{2}\right\rfloor}\left(R_{\Delta}\right)<0$, see Example 7.5.

Finally, in Section 8 we highlight a connection to the literature, showing that a Gorenstein algebra defined via apolarity by Gondim and Zappalà [GZ18] is an Artinian reduction of $R_{\Delta}$ in characteristic zero. As a consequence, Theorem B applies to the algebras in [GZ18] as well. Moreover, we show that Theorem $\mathrm{C}$ also goes through the Artinian reduction, and thus provides a criterion to decide if the algebras studied by Gondim and Zappalà have a quadratic Gröbner basis. Finally, in Remark 8.5 we fix a previous characterization [GZ18, Theorem 3.5] of quadraticity for such algebras.

\section{Preliminaries}

2.1. Graded algebras and modules. Let us fix a field $\mathbb{F}$ and consider a standard graded $\mathbb{F}$-algebra $R=\mathbb{F}\left[x_{1}, \ldots, x_{n}\right] / I$, for some homogeneous ideal $I$. The Hilbert series of $R$ is a rational function of the form

$$
\operatorname{Hilb}(R, t)=\frac{\sum_{i=0}^{s} h_{i} t^{i}}{(1-t)^{d}}
$$

with $\sum_{i} h_{i} \neq 0$. The numerator of $\operatorname{Hilb}(R, t)$ expressed as above is the $h$-polynomial of $R$, and the integer sequence $h(R)=\left(h_{0}, \ldots, h_{s}\right)$ is the $h$-vector of $R$. In general, the entries of the $h$-vector can be either positive or negative.

If $M$ is a finitely generated graded module over $R$, we can consider its minimal graded free resolution. This is the unique (up to isomorphism of chain complexes) complex of free $R$-modules and degree zero maps

$$
\cdots \stackrel{\partial_{i+1}}{\rightarrow} \bigoplus_{j} R(-j)^{\beta_{i, j}} \stackrel{\partial_{i}}{\rightarrow} \cdots \stackrel{\partial_{2}}{\rightarrow} \bigoplus_{j} R(-j)^{\beta_{1, j}} \stackrel{\partial_{1}}{\rightarrow} \bigoplus_{j} R(-j)^{\beta_{0, j}} \rightarrow 0
$$


which is exact in all positions but the zeroth, where $\operatorname{coker}\left(\partial_{1}\right) \cong M$, and is such that every $\partial_{i}$ can be represented as a matrix of zeros and homogeneous polynomials of positive degree. The numbers $\beta_{i, j}$ in (2.1) are known as the graded Betti numbers of $M$. In a more functorial fashion, they can be written as $\beta_{i, j}=\beta_{i, j}^{R}(M)=\operatorname{dim}_{\mathbb{F}} \operatorname{Tor}_{i}^{R}(M, \mathbb{F})_{j}$. This highlights a second way to compute graded Betti numbers: namely, thanks to the commutativity of Tor, one can tensor a minimal resolution of the $R$-module $\mathbb{F}$ with $M$, and then take the homology of the resulting chain complex. We will use this standard observation in the next sections. The quantity $\sup \left\{j-i: \beta_{i, j}^{R}(M) \neq 0\right\}$ is known as the Castelnuovo-Mumford regularity of $M$, denoted by $\operatorname{reg}_{R}(M)$.

The standard tool to record the information about graded Betti numbers is the Poincaré series of $M$ over $R$, defined as

$$
P_{R}^{M}(s, t)=\sum_{i, j} \beta_{i, j}^{R}(M) s^{j} t^{i}
$$

We will omit the superscript when $M$ is the residue field $\mathbb{F}=R /\left(x_{1}, \ldots, x_{n}\right)$.

If $M$ is generated in a single degree, we say that the minimal resolution of $M$ is linear for 0 steps; if moreover all the nonzero entries of the matrices $\partial_{i}$ in (2.1) are linear forms for every $1 \leq i \leq k$, we say that the minimal resolution of $M$ is linear for $k$ steps. If the nonzero entries of all matrices in the resolution are linear forms, we say that $M$ has a linear resolution.

By a celebrated theorem of Hilbert, minimal resolutions of modules over the polynomial ring $S=\mathbb{F}\left[x_{1}, \ldots, x_{n}\right]$ are finite: the length of the minimal free resolution of $M$ as an $S$-module is known as the projective dimension of $M$ and denoted by $\operatorname{pd}_{S}(M)$. When $M=R$, it is known that $n-d \leq \operatorname{pd}_{S}(R) \leq n$, where $d$ is the Krull dimension of $R$. Algebras attaining the above lower bound are well studied:

Definition 2.1. A $d$-dimensional standard graded $\mathbb{F}$-algebra $R=S / I$ is Cohen-Macaulay over $\mathbb{F}$ if $\operatorname{pd}_{S}(R)=n-d$.

Cohen-Macaulay algebras play an important role in commutative algebra, algebraic geometry and combinatorics [BH98, Sta96], and so does the special subclass consisting of Gorenstein algebras.

Definition 2.2. A $d$-dimensional standard graded $\mathbb{F}$-algebra $R=S / I$ is Gorenstein over $\mathbb{F}$ if it is Cohen-Macaulay and $\operatorname{dim}_{\mathbb{F}} \operatorname{Tor}_{n-d}^{S}(R, \mathbb{F})=1$.

We conclude this section by introducing an object that will play a crucial role in the rest of the paper.

Definition 2.3. Let $S=\mathbb{F}\left[x_{1}, \ldots, x_{n}\right]$ be a standard graded polynomial ring and let $R=S / I$ be a $d$-dimensional standard graded Cohen-Macaulay $\mathbb{F}$-algebra. Then:

- the canonical module of $S$ is the $\mathbb{Z}^{n}$-graded module $\omega_{S}:=S(-1,-1, \ldots,-1)$ (hence, if we are only interested in the $\mathbb{Z}$-graded structure, $\left.\omega_{S} \cong S(-n)\right)$;

- the canonical module of $R$ is the $\mathbb{Z}$-graded module $\omega_{R}:=\operatorname{Ext}_{S}^{n-d}\left(R, \omega_{S}\right)$. If $I$ happens to be $\mathbb{Z}^{n}$-graded, then so is $\omega_{R}$;

- the a-invariant of $R$ is $a(R):=-\min \left\{j \in \mathbb{Z}:\left(\omega_{R}\right)_{j} \neq 0\right\}$;

- the algebra $R$ is level if $\omega_{R}$ is generated in a single $\mathbb{Z}$-degree.

The minimal free resolution of $\omega_{R}$ as an $S$-module can be obtained dualizing a minimal free resolution of $R$ via the contravariant functor $\operatorname{Hom}_{S}\left(-, \omega_{S}\right)$. 
2.2. Simplicial complexes. Let $\Delta$ be a simplicial complex on $[n]=\{1, \ldots, n\}$, i.e., a collection of subsets of $[n]$ closed under inclusion. Elements of $\Delta$ are called faces and inclusion-maximal faces are called facets. We denote by $\mathcal{F}(\Delta)$ the set all facets of $\Delta$. The dimension af a face equals its cardinality minus one, and the dimension of $\Delta$ is the maximal dimension of one of its faces; if all facets have the same dimension, we say that $\Delta$ is pure. We record the number of faces in each dimension in a vector $f(\Delta)$ called the $f$-vector of $\Delta$. If $\Delta$ is $(d-1)$-dimensional, then $f(\Delta)=\left(f_{-1}, f_{0}, \ldots, f_{d-1}\right)$, where $f_{-1}=1$, unless $\Delta=\varnothing$. If $F_{1}, \ldots, F_{r}$ are subsets of $[n]$, we will denote by $\left\langle F_{1}, \ldots, F_{r}\right\rangle$ the simplicial complex generated by $F_{1}, \ldots, F_{r}$, i.e. the smallest simplicial complex on $[n]$ containing $F_{1}, \ldots, F_{r}$.

Fix now a field $\mathbb{F}$. We denote by $I_{\Delta} \subseteq \mathbb{F}\left[x_{1}, \ldots, x_{n}\right]$ the squarefree monomial ideal generated by the monomials supported on the complement of $\Delta$ in $2^{[n]}$, namely

$$
I_{\Delta}:=\left(\mathrm{x}^{F}: F \notin \Delta\right),
$$

where $\mathbf{x}^{F}=\prod_{i \in F} x_{i}$.

The ideal $I_{\Delta}$ is known as the Stanley-Reisner ideal of $\Delta$, and the graded $\mathbb{F}$-algebra $\mathbb{F}[\Delta]=$ $\mathbb{F}[\mathbf{x}] / I_{\Delta}$ is the associated Stanley-Reisner ring. There is a rich interplay between combinatorial properties of $\Delta$ and algebraic features of $\mathbb{F}[\Delta]$ : for instance, the Krull dimension of $\mathbb{F}[\Delta]$ is the dimension of $\Delta$ plus one. We can actually do better than this, as we can read off the whole $f$-vector of $\Delta$ from the Hilbert series of $\mathbb{F}[\Delta]$ and vice versa: the $h$-vector $h(\Delta)=h(\mathbb{F}[\Delta])=\left(h_{0}, \ldots, h_{d}\right)$ is an invertible integer linear transformation of $f(\Delta)$. More precisely:

$$
\begin{gathered}
h_{i}=\sum_{j=0}^{i}(-1)^{i-j}\left(\begin{array}{l}
d-j \\
d-i
\end{array}\right) f_{j-1} \\
f_{i-1}=\sum_{j=0}^{i}\left(\begin{array}{l}
d-j \\
d-i
\end{array}\right) h_{j} .
\end{gathered}
$$

Local properties of a simplicial complex around a face $F \in \Delta$ are described by the link of $F$, defined as

$$
\mathrm{lk}_{\Delta}(F):=\{G \in \Delta: F \cap G=\varnothing, F \cup G \in \Delta\} .
$$

If $\Delta$ is a pure $(d-1)$-dimensional complex, then $\operatorname{lk}_{\Delta}(F)$ is a (pure) $(d-|F|-1)$-dimensional simplicial complex. As a slogan, the simpler the homology of the links of $\Delta$ is, the nicer $\Delta$ is. To make this precise, we give the following definition after Murai and Terai [MT09] (even though it was already implicit in work by Terai and Yanagawa [Yan00]):

Definition 2.4. Let $r \geq 1$. A simplicial complex $\Delta$ satisfies the (combinatorial) Serre condition $\left(S_{r}\right)$ with respect to a field $\mathbb{F}$ if

$$
\widetilde{H}_{i}\left(\operatorname{lk}_{\Delta}(F) ; \mathbb{F}\right)=0
$$

for every $F \in \Delta$ and for every $i<\min \left\{r-1, \operatorname{dim}\left(\operatorname{lk}_{\Delta}(F)\right)\right\}$. A $(d-1)$-dimensional simplicial complex satisfying property $\left(S_{d}\right)$ over $\mathbb{F}$ is called Cohen-Macaulay over $\mathbb{F}$.

Definition 2.4 deserves some comments. It is clear that every complex $\Delta$ must satisfy $\left(S_{1}\right)$ and that, if $\Delta$ has $\left(S_{r}\right)$, then it has $\left(S_{i}\right)$ for every $1 \leq i \leq r$. Moreover, $\Delta$ satisfies property $\left(S_{2}\right)$ if and only if it is pure and the link of any face of codimension at least two is connected. In particular, property $\left(S_{2}\right)$ does not depend on the field. For $r \geq 2$, condition $\left(S_{r}\right)$ states that 
the link of any face of codimension at most $r$ is allowed to have nonvanishing homology only in top homological degree, while lower-dimensional faces need to have vanishing homology up to homological degree $r-2$. The combinatorial Serre condition $\left(S_{r}\right)$ for $\Delta$ (with respect to $\mathbb{F})$ is equivalent to the usual algebraic Serre condition $\left(S_{r}\right)$ for the Stanley-Reisner ring $\mathbb{F}[\Delta]$. In particular, a $(d-1)$-dimensional complex $\Delta$ is $\left(S_{d}\right)$ if and only if $\mathbb{F}[\Delta]$ satisfies the algebraic $\left(S_{d}\right)$ condition, which in turn means that $\mathbb{F}[\Delta]$ is a Cohen-Macaulay ring. This fact is known as Reisner's criterion [Rei76]. The equivalence between the algebraic and combinatorial Serre conditions for simplicial complexes is known and proved for instance in [Ter07, end of Section 1], but we will sketch a proof here for the interested reader.

Proposition 2.5. Let $r \geq 1$ and let $\Delta$ be a simplicial complex on $[n]$. Then $\Delta$ satisfies the combinatorial $\left(S_{r}\right)$ condition (with respect to $\mathbb{F}$ ) if and only if the Stanley-Reisner ring $\mathbb{F}[\Delta]$ satisfies the usual algebraic $\left(S_{r}\right)$ condition, i.e.

$$
\operatorname{depth} \mathbb{F}[\Delta]_{\mathfrak{p}} \geq \min \left\{r, \operatorname{dim} \mathbb{F}[\Delta]_{\mathfrak{p}}\right\} \quad \text { for every } \mathfrak{p} \in \operatorname{Spec}(\mathbb{F}[\Delta])
$$

Proof: For a Noetherian ring $R$, the algebraic $\left(S_{1}\right)$ condition is known to be equivalent to the absence of embedded primes in $R$, which is always the case for the reduced ring $\mathbb{F}[\Delta]$. On the other hand, the combinatorial $\left(S_{1}\right)$ condition is met by every simplicial complex.

Let now $r \geq 2$, and denote by $S$ the polynomial ring $\mathbb{F}\left[x_{1}, \ldots, x_{n}\right]$. Note that both the combinatorial and the algebraic $\left(S_{2}\right)$ conditions imply that $\mathbb{F}[\Delta]$ is equidimensional, i.e. $\Delta$ is pure: for the algebraic statement, see [Har62, Remark 2.4.1]. By [DMV19, Proposition 2.11], (2.5) is then equivalent to

$$
\operatorname{dim} \operatorname{Ext}_{S}^{n-j}\left(\mathbb{F}[\Delta], \omega_{S}\right) \leq j-r \quad \text { for every } j<\operatorname{dim} \mathbb{F}[\Delta]
$$

which, since $\operatorname{Ext}_{S}^{n-j}\left(\mathbb{F}[\Delta], \omega_{S}\right)$ is a squarefree module [Yan00], is in turn equivalent to

$$
\operatorname{Ext}_{S}^{n-j}\left(\mathbb{F}[\Delta], \omega_{S}\right)_{F}=0 \quad \text { for every } j<\operatorname{dim}(\Delta)+1 \text { and } F \in\{0,1\}^{n} \text { with }|F|>j-r .
$$

It is a useful fact of Stanley-Reisner theory that $\operatorname{Ext}_{S}^{n-j}\left(\mathbb{F}[\Delta], \omega_{S}\right)_{F} \cong H_{j-|F|-1}\left(\operatorname{lk}_{\Delta} F, \mathbb{F}\right)$ : see for instance [Yan00, Proposition 3.1]. To conclude it is then enough to set $i=j-|F|-1$ and note that, since $\Delta$ is pure, $\operatorname{dim} \mathrm{lk}_{\Delta} F=\operatorname{dim} \Delta-|F|$.

The Alexander dual of $\Delta$ is the simplicial complex $\Delta^{*}$ on the same vertex set as $\Delta$ and with Stanley-Reisner ideal

$$
I_{\Delta^{*}}=\left(\mathbf{x}^{[n] \backslash G}: G \in \Delta\right),
$$

where $\mathbf{x}^{[n] \backslash G}=\prod_{i \in[n] \backslash G} x_{i}$. A minimal generating set for $I_{\Delta^{*}}$ is hence given by monomials supported on the complements of facets of $\Delta$ in $[n]$.

Eagon and Reiner [ER98] related the Cohen-Macaulay property of $\Delta$ to the CastelnuovoMumford regularity of the Alexander dual ideal $I_{\Delta^{*}}$. This result was extended to $\left(S_{r}\right)$ conditions by Terai and Yanagawa [Yan00].

Theorem 2.6. [ER98, Theorem 3], [Yan00, Corollary 3.7] Let $\Delta$ be a $(d-1)$-dimensional simplicial complex on $[n]$. For every $2 \leq r \leq d$, the complex $\Delta$ satisfies property $\left(S_{r}\right)$ if and only if $I_{\Delta^{*}}$ has a resolution as an $\mathbb{F}\left[x_{1}, \ldots, x_{n}\right]$-module which is linear for $r-1$ steps. In particular, $\Delta$ is Cohen-Macaulay if and only if $I_{\Delta^{*}}$ has a linear $\mathbb{F}\left[x_{1}, \ldots, x_{n}\right]$-resolution. 
2.3. Nagata idealization. A theme of this paper will be the construction of Gorenstein algebras from "good enough" objects. The required technical tool is a well-known operation called idealization, made popular by Nagata. This subsection follows closely [MSS21a, Section 3], and we refer the reader to that for more information.

Definition 2.7. The idealization of an $A$-module $M$, denoted $A \ltimes M$, is the $A$-algebra with underlying module $A \oplus M$ and multiplication defined by $\left(a_{1}, m_{1}\right) \cdot\left(a_{2}, m_{2}\right)=\left(a_{1} a_{2}, a_{1} m_{2}+\right.$ $\left.a_{2} m_{1}\right)$.

This operation is called idealization because it turns every submodule $N$ of $M$ into an ideal $\{0\} \ltimes N$ of $A \ltimes M$. For the rest of the paper, the $\operatorname{ring} A$ will be a standard graded $\mathbb{F}$-algebra. If $M$ is graded, then so is $A \ltimes M$, by setting $(A \ltimes M)_{j}=A_{j} \oplus M_{j}$; moreover, $A \ltimes M$ is standard graded if and only if $M$ is generated in degree one [MSS21a, Remark 3.1]. Note that, if $A$ and $M$ are $\mathbb{Z}^{n}$-graded, then $A \ltimes M$ inherits the $\mathbb{Z}^{n}$-grading.

We record here for later use a lemma that relates the homological information of an idealization to that of its building blocks.

Lemma 2.8. [Gul72, Theorem 2] Let $A$ be a standard graded $\mathbb{F}$-algebra and let $M$ be a finitely generated graded $A$-module generated in degree one. Then

$$
P_{A \ltimes M}(s, t)=\frac{P_{A}(s, t)}{1-t P_{A}^{M}(s, t)},
$$

where $P_{A}^{M}(s, t)$ is the Poincaré series of $M$ as an $A$-module, as defined in (2.2).

Definition 2.9. A standard graded Cohen-Macaulay $\mathbb{F}$-algebra $A$ is superlevel if it is level and its canonical module $\omega_{A}$ has a linear presentation as an $A$-module, i.e., there is an exact sequence

$$
A(a-1)^{b} \stackrel{\varphi_{1}}{\rightarrow} A(a)^{g} \stackrel{\varphi_{0}}{\rightarrow} \omega_{A} \rightarrow 0
$$

with $a=a(A)$ as in Definition 2.3.

We end this subsection by collecting several results proved in [MSS21a, Section 3]. Parts iv and $\mathrm{v}$ follow immediately by analyzing the graded structure of the idealization and using the formula for the Hilbert series of the canonical module [BH98, Corollary 4.4.6].

Theorem 2.10 ([MSS21a, Proposition 3.2, Lemma 3.3]). Let $A=S / I$ be a standard graded and level $\mathbb{F}$-algebra and let $\widetilde{A}:=A \ltimes \omega_{A}(-a(A)-1)$. The following statements hold:

i. $\widetilde{A}$ is a standard graded and Gorenstein $\mathbb{F}$-algebra;

ii. if $\omega_{A}$ is minimally generated by $g$ elements, then

$$
\widetilde{A}=\frac{S\left[z_{1}, \ldots z_{g}\right]}{I+\mathcal{L}+\left(z_{1}, \ldots, z_{g}\right)^{2}},
$$

where

$$
\mathcal{L}:=\left(\sum_{i=1}^{g} f_{i} z_{i}:\left(f_{1}, \ldots, f_{g}\right) \in \operatorname{Syz}_{1}^{S}\left(\omega_{A}\right)\right) ;
$$

iii. if $A$ is quadratic, then $\widetilde{A}$ is quadratic if and only if $A$ is superlevel.

iv. $h_{i}(\widetilde{A})=h_{i}(A)+h_{s-i+1}(A)$ for every $1<i<s-1$.

v. $\operatorname{dim}(\widetilde{A})=\operatorname{dim}(A)$. 
2.4. Koszul algebras. The last ingredient we need is a special class of standard graded algebras called Koszul algebras: for a survey, we direct the reader to [CDNR13].

Definition 2.11. A standard graded $\mathbb{F}$-algebra $A$ is $K$ oszul (over $\mathbb{F}$ ) if $\mathbb{F}$ has a linear resolution as an $A$-module.

In the above definition, $\mathbb{F}$ is identified with the quotient of $A$ by its maximal homogeneous ideal. Observe that, by definition, $A$ is Koszul if and only if $P_{A}(s, t) \in \mathbb{Z}[[s t]]$.

Due to the fact that a minimal resolution of $\mathbb{F}$ as an $A$-module is typically infinite, it is in general very hard to prove that a certain algebra $A$ is Koszul. However, the following is well-known.

Proposition 2.12. Let $A=\mathbb{F}\left[x_{1}, \ldots, x_{n}\right] / I$ be a standard graded $\mathbb{F}$-algebra and assume without loss of generality that $I \subseteq\left(x_{1}, \ldots, x_{n}\right)^{2}$. Then:

- if $A$ is Koszul, then I is generated in degree 2;

- if I has a Gröbner basis of quadrics, then A is Koszul.

Having a Gröbner basis of quadrics is actually a much stronger condition than Koszulness, and we will see in Section 6 how this translates combinatorially for the algebras studied in the present paper.

We close this section by a statement highlighting how Koszulness interacts with suitable idealizations.

Lemma 2.13. Let $A$ be a Koszul $\mathbb{F}$-algebra and let $M$ be a finitely generated graded $A$-module generated in degree one. Then the residue field $\mathbb{F}$ has an $(A \ltimes M)$-resolution which is linear for $k$ steps if and only if the A-module $M$ has a resolution which is linear for $k-1$ steps. In particular, $A \ltimes M$ is Koszul if and only if $M$ has a linear A-resolution.

Proof: The statement follows from comparing the coefficients in (2.6). Since $A$ is Koszul, one has that $P_{A}(s, t) \in \mathbb{Z}[[s t]]$. Let $P_{A \ltimes M}(s, t)=\sum_{i, j} b_{i, j} s^{j} t^{i}$ and $P_{A}^{M}(s, t)=\sum_{i, j} c_{i, j} s^{j} t^{i}$. After multiplying both sides of (2.6) by $1-t P_{A}^{M}(s, t)$, we analyze the coefficient of $s^{j} t^{i}$ for every $i, j$ with $i<j$, obtaining that

$$
b_{i, j}-\sum_{h, \ell} b_{h, \ell} c_{i-h-1, j-\ell}=0 .
$$

Note that $b_{0,0}=1$ and $b_{0, j}=0$ for every $j>0$. We want to show that

$$
b_{i, j}=0 \text { for every } 1 \leq i \leq k, j>i \Leftrightarrow c_{i-1, j}=0 \text { for every } 1 \leq i \leq k, j>i .
$$

To prove the left to right arrow it is enough to observe that, for every $1 \leq i \leq k$ and $j>i$, (2.9) implies that $0=b_{i, j} \geq b_{0,0} c_{i-1, j}=c_{i-1, j} \geq 0$.

Conversely, let us now assume that $c_{i-1, j}=0$ for every $1 \leq i \leq k$ and $j>i$, and assume by contradiction that there exists $1 \leq m \leq k$ such that $b_{m, q}>0$ for some $q>m$. We can then pick this $m$ to be the smallest possible with respect to this property. Because of (2.9), it holds that $b_{m, q}=\sum_{h, \ell} b_{h, \ell} c_{m-h-1, q-\ell}$. Since every $h$ in the sum must be strictly smaller than $m$, it follows that $b_{h, \ell}$ vanishes whenever $h<\ell$. Hence, $0<b_{m, q}=\sum_{h} b_{h, h} c_{m-h-1, q-h}$, and hence there must be at least one nonvanishing $c_{m-h-1, q-h}$, contradicting the hypothesis. 


\section{Bier BALls AND SPHERES}

In this section we recall a simple construction which associates with any simplicial complex on $[n]$ (other than the full simplex) an (n-1)-dimensional PL ball which is a proper subcomplex of the boundary complex of the $n$-dimensional cross-polytope. Such complexes were notably studied in higher generality by Murai [Mur11].

Definition 3.1. Let $\Delta$ be a simplicial complex on $n$ vertices, and let $I_{\Delta} \subseteq \mathbb{F}[\mathbf{x}]$ be its Stanley-Reisner ideal. We define a new complex $\Gamma$ on $2 n$ vertices by

$$
I_{\Gamma}:=I_{\Delta}+\left(x_{i} y_{i}: i \in[n]\right) \subseteq \mathbb{F}[\mathbf{x}, \mathbf{y}] .
$$

If $\Delta$ is not the $(n-1)$-dimensional simplex, we will call $\Gamma$ the Bier ball associated with $\Delta$ : see Proposition 3.4 below.

Remark 3.2. Most of the extant literature does not really deal with Bier balls, but rather with the PL spheres that bound them: these are known as Bier spheres after Thomas Bier, who introduced them in [Bie92] (see also [BPSZ05, DFN19]).

Remark 3.3. For $\Delta$ flag, the construction in Definition 3.1 has already appeared in the literature several times, notably in [CV13, Proposition 4.1]. The earliest reference we are aware of is [Vil90, Proposition 2.2], which has a slightly different take on the subject. Indeed, if $\Delta$ is flag, the ideal $I_{\Delta}$ can be seen as the edge ideal of a graph $G$, and $I_{\Gamma}$ becomes the edge ideal of the graph obtained from $G$ by adding extra leaves (or "whiskers") to every vertex.

Proposition 3.4. Let $\Delta$ be a simplicial complex on $[n]$ and let $\Gamma$ be as in Definition 3.1. Then:

i. $\Gamma$ is a shellable $(n-1)$-dimensional simplicial complex;

ii. $h(\Gamma)=f(\Delta)$;

iii. if $\Delta$ is not the $(n-1)$-simplex, then $\Gamma$ is a $P L(n-1)$-ball and its boundary complex $\partial \Gamma$ is the $P L(n-2)$-sphere known as the Bier sphere associated with $\Delta$ [Bie92];

iv. the canonical module $\omega_{\mathbb{F}[\Gamma]}$ is isomorphic as an $\mathbb{F}[\Gamma]$-module to the ideal of $\mathbb{F}[\Gamma]$ generated by the monomials $\mathbf{y}^{[n] \backslash F}$, where $F$ ranges over all facets of $\Delta$ (here $\mathbf{y}^{\varnothing}$ is equal to 1). In other words, the canonical module of $\mathbb{F}[\Gamma]$ is the image of the Alexander dual ideal of $\Delta$ (in the y-variables) under the projection $\mathbb{F}[\mathbf{x}, \mathbf{y}] \rightarrow \mathbb{F}[\Gamma]$.

We note that Proposition 3.4 was essentially already proved in [Mur11, Lemma 1.4, Theorem 1.14 and Theorem 3.6], where a more general construction associating a Bier ball with every multicomplex is studied. However, we will sketch our approach below for the reader's benefit. First of all, note that the facets of $\Gamma$ are precisely the sets $F^{\sharp}=\left\{x_{i} \mid i \in F\right\} \cup\left\{y_{j} \mid j \notin F\right\}$, where $F$ ranges over all faces of $\Delta$. Order (partially) the facets of $\Gamma$ so that $F \sharp<G^{\sharp}$ when $\operatorname{dim}(F)<\operatorname{dim}(G)$. It is left to the reader to show that any total order refining $<$ gives a shelling order for $\Gamma$ (for the definition of a shelling order, see Definition 6.1). Moreover, since the minimal new face we are adding at each step of the shelling has cardinality $|F|$, the claim about the $h$-vector of $\mathbb{F}[\Gamma]$ follows from [Sta96, Proposition III.2.3].

Now note that every face of $\Gamma$ of codimension 1 is contained in at most two facets. Indeed, every codimension 1 face is of the form $F_{\sharp} \backslash\left\{v_{i}\right\}$, where $i \in[n]$ and $v_{i}$ is either $x_{i}$ or $y_{i}$. As $\left\{x_{j}, y_{j}\right\}$ is a nonface of $\Gamma$ for every $j \in[n]$, one can extend the given codimension 1 face to a facet only by adding either $y_{i}$ (which always yields a facet) or $x_{i}$ (which might yield a facet, 
depending on $\Delta$ ). By [Bjö95, Theorem 11.4], the shellable complex $\Gamma$ must then be either a PL ball or a PL sphere, with the former case occurring when there exists a codimension 1 face contained in exactly one facet. This happens whenever $\Delta$ has at least a missing face, i.e., it is not the full $(n-1)$-simplex.

Finally, the last statement follows from the description of the canonical module of any homology ball (see for instance [BH98, Theorem 5.7.1]), which says that $\omega_{\mathbb{F}[\Gamma]}$ is the image of $I_{\partial \Gamma}$ under the projection $\mathbb{F}[\mathbf{x}, \mathbf{y}] \rightarrow \mathbb{F}[\Gamma]$. Recalling that $\partial \Delta$ consists of the codimension 1 faces of $\Gamma$ contained in exactly one facet, one checks that $I_{\partial \Gamma}=I_{\Gamma}+\left(\mathbf{y}^{[n] \backslash F} \mid F \in \mathcal{F}(\Delta)\right)$. This is precisely the definition of a Bier sphere, see for instance [DFN19, Corollary 5.3]. Note that

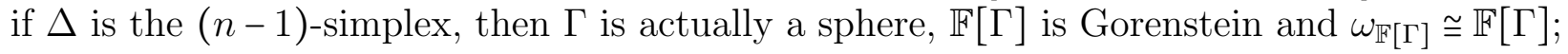
since $\mathbf{y}^{\varnothing}=1$, we are done also in this case.

The simplicial complex $\Gamma$ is actually vertex decomposable [Mur11, Remark 1.8], a property which is stronger than shellability. Moreover, any Bier $(n-1)$-ball or $(n-2)$-sphere is naturally a subcomplex of the boundary complex $\diamond_{n}$ of the $n$-cross-polytope, with $I_{\diamond_{n}}=$ $\left(x_{i} y_{i}: i \in[n]\right)$.

Example 3.5. Let $n=3$ and $\Delta$ be the simplicial complex $\left\{\varnothing, x_{1}, x_{2}, x_{3}, x_{1} x_{2}\right\}$. Then $I_{\Gamma}=$ $\left(x_{1} x_{3}, x_{2} x_{3}\right)+\left(x_{1} y_{1}, x_{2} y_{2}, x_{3} y_{3}\right)$, and $\Gamma$ is the 2 -dimensional pure shellable homology ball with shelling order

$$
y_{1} y_{2} y_{3}<\underline{x_{1}} y_{2} y_{3}<y_{1} \underline{x_{2}} y_{3}<y_{1} y_{2} \underline{x_{3}}<\underline{x_{1} x_{2}} y_{3} .
$$

Observe that $h(\Gamma)=f(\Delta)=(1,3,1)$. The complex $\partial \Gamma=\left\langle x_{1} x_{2}, x_{2} y_{1}, y_{1} x_{3}, y_{2} x_{3}, x_{1} y_{2}\right\rangle$ is a 5 -cycle.
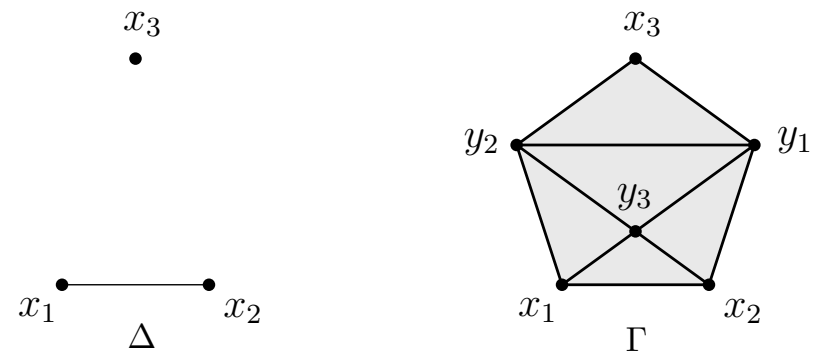

Figure 1. The simplicial complex $\Delta$ as in Example 3.5 and the associated Bier ball $\Gamma$.

Remark 3.6. The Bier sphere $\partial \Gamma$ in Example 3.5 is flag, but this is usually not the case in general. In [HK12], the authors prove that there are only 8 possible complexes $\Delta$ which give rise to flag Bier spheres.

\section{From Bier balls to Gorenstein algebras: the Ring $R_{\Delta}$}

For the rest of this article we will be concerned with idealizing the canonical module of the Stanley-Reisner ring of a Bier ball. We set some notation for the rest of the paper.

Notation 4.1. From now on, if not specified otherwise:

- $\Delta$ is a pure $(d-1)$-dimensional simplicial complex on $n$ vertices, and its StanleyReisner ideal $I_{\Delta}$ lives in $\mathbb{F}\left[x_{1}, \ldots, x_{n}\right]$; 
- $S$ is the polynomial ring $\mathbb{F}[\mathbf{x}, \mathbf{y}]=\mathbb{F}\left[x_{1}, \ldots, x_{n}, y_{1}, \ldots, y_{n}\right]$;

- $\Gamma$ is the $(n-1)$-dimensional Bier ball obtained from $\Delta$ as in Definition 3.1 (with a slight abuse of notation, we will use the name "Bier ball" also when $\Delta$ is the full simplex);

- $R_{\Delta}$ is the $n$-dimensional standard graded Gorenstein $\mathbb{F}$-algebra $\widetilde{\mathbb{F}[\Gamma]}=\mathbb{F}[\Gamma] \ltimes \omega_{\mathbb{F}[\Gamma]}(n-$ $d-1$ ), as in Theorem 2.10 (note that, by Proposition 3.4.iv, $\mathbb{F}[\Gamma]$ is level and $a(\mathbb{F}[\Gamma])=$ $-(n-d)=d-n)$

- given two facets $F_{1}$ and $F_{2}$ of $\Delta$, we will denote by $b_{F_{1}, F_{2}}$ the binomial $\mathbf{y}^{F_{1} \backslash F_{2}} z_{F_{1}}$ $\mathbf{y}^{F_{2} \backslash F_{1}} z_{F_{2}}$ inside $S\left[z_{F} \mid F \in \mathcal{F}(\Delta)\right]$. Notice that $b_{F_{2}, F_{1}}=-b_{F_{1}, F_{2}}$, and the case where $F_{1}$ and $F_{2}$ coincide yields the zero binomial.

Remark 4.2. The construction of $R_{\Delta}$ shows that if $f=\left(1, f_{0}, \ldots, f_{d-1}\right)$ is the $f$-vector of a pure simplicial complex, then $\left(1, f_{0}+f_{d-1}, f_{1}+f_{d-2}, \ldots, f_{d-1}+f_{0}, 1\right)$ is the $h$-vector of a Gorenstein standard graded algebra. In particular, it is an $M$-sequence (see [Sta96]). We will see a stronger condition on $\Delta$ for this vector to be the the $h$-vector of a quadratic - or even Koszul - Gorenstein algebra.

Proposition 4.3. Let $\Delta$ be a pure simplicial complex of dimension $d-1$. Then

$$
R_{\Delta}=\frac{S\left[z_{F} \mid F \in \mathcal{F}(\Delta)\right]}{I_{\Gamma}+\mathcal{L}+\left(z_{F} \mid F \in \mathcal{F}(\Delta)\right)^{2}},
$$

where

$$
\mathcal{L}=\left(x_{i} z_{F} \mid F \in \mathcal{F}(\Delta), i \notin F\right)+\left(b_{F_{1}, F_{2}} \mid F_{1}, F_{2} \in \mathcal{F}(\Delta), F_{1} \neq F_{2}\right) .
$$

In particular, the defining ideal of $R_{\Delta}$ is generated by

- $\mathbf{x}^{N_{i}}$, where $N_{i}$ is a minimal nonface of $\Delta$,

- $x_{i} y_{i}$, where $i$ ranges between 1 and $n$,

- $z_{F_{1}} z_{F_{2}}$, where $F_{1}$ and $F_{2}$ are (possibly coincident) facets of $\Delta$,

- $x_{i} z_{F}$, where $F$ is a facet of $\Delta$ and $i \notin F$,

- $b_{F_{1}, F_{2}}$, where $F_{1}$ and $F_{2}$ are distinct facets of $\Delta$ and $b_{F_{1}, F_{2}}$ is the binomial defined in Notation 4.1,

and such a presentation does not depend on the characteristic of the field $\mathbb{F}$.

Proof: Since the defining ideal of the claimed presentation of $R_{\Delta}$ involves only monomials and binomials of the form $\mathbf{m}-\mathbf{m}^{\prime}$, such a presentation must hold in every characteristic, see e.g. [D'A17, Lemma A.1.(iii)].

By Theorem 2.10.ii, in order to find a presentation for $R_{\Delta}$ it is enough to compute the syzygies of $\omega_{\mathbb{F}[\Gamma]}$ as an $S$-module. (Since we are quotienting by $I_{\Gamma}$ in (2.7), we are actually investigating the $\mathbb{F}[\Gamma]$-syzygies of $\omega_{\mathbb{F}[\Gamma]}$, as $\operatorname{Syz}_{1}^{\mathbb{F}[\Gamma]}\left(\omega_{\mathbb{F}[\Gamma]}\right) \cong \operatorname{Syz}_{1}^{S}\left(\omega_{\mathbb{F}[\Gamma]}\right) / I_{\Gamma} \operatorname{Syz}_{1}^{S}\left(\omega_{\mathbb{F}[\Gamma]}\right)$.) By Proposition 3.4.iv and by the usual Alexander duality (see e.g. [MS05, Proposition 1.37]), the canonical module $\omega_{\mathbb{F}[\Gamma]}$ can be identified with the monomial ideal of $\mathbb{F}[\Gamma]=S / I_{\Gamma}$ generated by $\left\{\overline{\mathbf{y}^{F^{c}}}: F\right.$ facet of $\left.\Delta\right\}$. Here and until the end of the proof, we will use the shorthand $F^{c}$ to denote $[n] \backslash F$, the complement of $F$, while the overline denotes the class of $\mathbf{y}^{F^{c}}$ in $\mathbb{F}[\Gamma]$. Hence, the purity of $\Delta$ implies that all such generators have the same degree $n-d$. Calling 
$F_{1}, \ldots, F_{r}$ the facets of $\Delta$, our task is to compute the kernel of the map of $S$-modules

$$
\begin{aligned}
S(-n+d)^{|\mathcal{F}(\Delta)|} & \rightarrow S / I_{\Gamma}, \\
\mathbf{1}_{F_{i}} & \mapsto \overline{\mathbf{y}^{F_{i}^{c}}},
\end{aligned}
$$

where each $\mathbf{1}_{F_{i}}$ has multidegree $F_{i}^{c}$. To do so, we follow [GP08, Remark 2.5.6]: let $N_{1}, \ldots, N_{s}$ be the minimal nonfaces of $\Delta$ and let $A$ be the 1-by- $(r+n+s)$ matrix

$$
A=\left(\mathbf{y}^{F_{1}^{c}} \ldots \mathbf{y}^{F_{r}^{c}}\left|x_{1} y_{1} \ldots x_{n} y_{n}\right| \mathbf{x}^{N_{1}} \ldots \mathbf{x}^{N_{s}}\right) .
$$

Ignoring the degrees, the matrix $A$ represents a map $S^{r+n+s} \rightarrow S$. The $S$-module obtained by projecting the syzygy module $\operatorname{Syz}^{S}(A)$ on its first $r$ coordinates is precisely $\operatorname{Syz}_{1}^{S}\left(\omega_{\mathbb{F}[\Gamma]}\right)$. Since all entries of $A$ are monomials, Schreyer's theorem [Eis95, Theorem 15.10] implies that computing syzygies between such monomials is enough to seize $\operatorname{Syz}^{S}(A)$ and hence $\operatorname{Syz}_{1}^{S}\left(\omega_{\mathbb{F}[\Gamma]}\right)$. Let $a$ and $b$ be two of the entries in $A$. If $a$ and $b$ both belong to $I_{\Gamma}$, the syzygy between them will live in the last $n+s$ components of $S^{r+n+s}$ and will hence not matter for our final aim. From now on, assume thus that $a=\mathbf{y}_{i}^{F_{i}^{c}}$ for some $i$.

- If $b \in I_{\Gamma}$ and $\operatorname{gcd}(a, b)=1$, then the syzygy between $a$ and $b$ will vanish after projecting and taking the quotient modulo $I_{\Gamma}$. In particular, this syzygy will not be involved in the presentation of $R_{\Delta}$.

- If $b \in I_{\Gamma}$ and $\operatorname{gcd}(a, b)>1$, then it must be that $b=x_{j} y_{j}$ and $y_{j}$ divides $\mathbf{y}^{F_{i}^{c}}$, i.e. $j \notin F_{i}$. This gives rise to the syzygy $x_{j} \mathbf{1}_{F_{i}}$.

- Finally, assume $b=\mathbf{y}_{j}^{F_{j}^{c}}$ for some $j \neq i$. Then, noting that $F_{j}^{c} \backslash F_{i}^{c}=F_{i} \backslash F_{j}$, we obtain the syzygy $\mathbf{y}^{F_{i} \backslash F_{j}} \mathbf{1}_{F_{i}}-\mathbf{y}^{F_{j} \backslash F_{i}} \mathbf{1}_{F_{j}}$ and this ends the proof.

As an application, we determine when $R_{\Delta}$ is quadratic.

Proposition 4.4. Let $\Delta$ be a pure flag simplicial complex. Then $R_{\Delta}$ is quadratic if and only if $\Delta$ is $\left(S_{2}\right)$.

Proof: Proceeding as in the proof of Proposition 4.3, one sees that $\omega_{\mathbb{F}[\Gamma]}$ has first linear $\mathbb{F}[\Gamma]$-syzygies if and only if the Alexander dual ideal of $\Delta$ has first linear $\mathbb{F}[\mathbf{y}]$-syzygies. By Theorem 2.6, this happens precisely when $\Delta$ is $\left(S_{2}\right)$.

Proposition 4.4 will be vastly generalized in the next section: see Theorem 5.1.

Proposition 4.5. The collection $\mathcal{U}$ of monomials and binomials listed in Proposition 4.3 is a universal Gröbner basis for the ideal defining $R_{\Delta}$ in any characteristic.

Proof: $\quad$ Proposition 4.3 says that the defining ideal of $R_{\Delta}$ admits a generating set $\mathcal{G}$ consisting of monomials and binomials of the form $\mathbf{m}-\mathbf{m}^{\prime}$. Any Gröbner basis obtained by applying Buchberger's algorithm to the generating set $\mathcal{U}$ will have the same property, and will hence be characteristic-independent.

It is then enough to show that, for any term order <, any possible $S$-polynomial reduces to zero with respect to $\mathcal{U}$. The only interesting situation arises when we consider the $S$ polynomial of two distinct binomials $b$ and $b^{\prime}$, as all the other generators are monomial. If the leading terms of $b$ and $b^{\prime}$ do not share the same $z$-variable, then $S\left(b, b^{\prime}\right)$ has degree two in the $z$-variables and hence reduces to zero with respect to $\mathcal{U}$. 
We can hence assume that $b=b_{F, G_{1}}, b^{\prime}=b_{F, G_{2}}, \operatorname{in}_{<}\left(b_{F, G_{1}}\right)=\mathbf{y}^{F \backslash G_{1}} z_{F}$ and $\operatorname{in}_{<}\left(b_{F, G_{2}}\right)=\mathbf{y}^{F \backslash G_{2}} z_{F}$ (where $F, G_{1}$ and $G_{2}$ are three distinct facets of $\Delta$ ).

We then get that

$$
\begin{aligned}
S\left(b, b^{\prime}\right) & =-\mathbf{y}^{\left(F \backslash G_{2}\right) \backslash\left(F \backslash G_{1}\right)} \mathbf{y}^{G_{1} \backslash F} z_{G_{1}}+\mathbf{y}^{\left(F \backslash G_{1}\right) \backslash\left(F \backslash G_{2}\right)} \mathbf{y}^{G_{2} \backslash F} z_{G_{2}} \\
& =-\mathbf{y}^{\left(F \backslash G_{2}\right) \cap G_{1}} \mathbf{y}^{G_{1} \backslash F} z_{G_{1}}+\mathbf{y}^{\left(F \backslash G_{1}\right) \cap G_{2}} \mathbf{y}^{G_{2} \backslash F} z_{G_{2}} \\
& =\mathbf{y}^{\left(G_{1} \cap G_{2}\right) \backslash F} b_{G_{2}, G_{1}}
\end{aligned}
$$

and, since $b_{G_{2}, G_{1}} \in \mathcal{U}$, we are done.

Example 4.6. Let $\Delta$ be the pure flag simplicial complex with facet list $\{123,234,345\}$. The binomials arising from comparing facets of $\Delta$ are

$$
\begin{aligned}
& b_{123,234}=y_{1} z_{123}-y_{4} z_{234}, \\
& b_{234,345}=y_{2} z_{234}-y_{5} z_{345}, \\
& b_{123,345}=y_{1} y_{2} z_{123}-y_{4} y_{5} z_{345} .
\end{aligned}
$$

However, the binomial $b_{123,345}$ is redundant, since it can be written as $y_{2} b_{123,234}+y_{4} b_{234,345}$. Therefore, $R_{\Delta}$ can be presented as the quotient of $\mathbb{F}\left[x_{1}, \ldots, x_{5}, y_{1}, \ldots, y_{5}, z_{123}, z_{234}, z_{345}\right]$ by the ideal

$$
\begin{aligned}
& \left(x_{1} x_{4}, x_{1} x_{5}, x_{2} x_{5}, x_{1} y_{1}, x_{2} y_{2}, x_{3} y_{3}, x_{4} y_{4}, x_{5} y_{5}, z_{123}^{2}, z_{234}^{2}, z_{345}^{2},\right. \\
& z_{123} z_{234}, z_{123} z_{345}, z_{234} z_{345}, x_{4} z_{123}, x_{5} z_{123}, x_{1} z_{234}, x_{5} z_{234}, x_{1} z_{345}, x_{2} z_{345}, \\
& \left.y_{4} z_{234}-y_{1} z_{123}, y_{5} z_{345}-y_{2} z_{234}\right) .
\end{aligned}
$$

Note this agrees with Proposition 4.4: being Cohen-Macaulay, $\Delta$ is also $\left(S_{2}\right)$.

\section{When is $R_{\Delta}$ Koszul?}

We now wish to investigate the Koszul property of the $\mathbb{F}$-algebra $R_{\Delta}$. The main result, whose proof is postponed to the end of the section, is the following:

Theorem 5.1. Let $\Delta$ be a flag $(d-1)$-dimensional simplicial complex and let $1 \leq k \leq d$. $\mathbb{F}$ has a resolution as an $R_{\Delta}$-module which is linear for $k$ steps if and only if $\Delta$ satisfies property $\left(S_{k}\right)$. Moreover, if $\mathbb{F}$ has an $R_{\Delta}$-resolution which is linear for d steps, then it has a linear $R_{\Delta}$-resolution.

Remark 5.2. The homological behaviour of $R_{\Delta}$ is especially remarkable, since for a general standard graded quadratic $\mathbb{F}$-algebra $Q$ the linearity of the $Q$-resolution of $\mathbb{F}$ might fail at an arbitrarily high homological position [Roo93].

As a corollary of Theorem 5.1 we obtain the following:

Corollary 5.3. Let $\Delta$ be a flag simplicial complex. Then $R_{\Delta}$ is Koszul over $\mathbb{F}$ if and only if $\Delta$ is Cohen-Macaulay over $\mathbb{F}$. 
5.1. The generalized Koszul complex for a quadratic Stanley-Reisner ring. Let $\Sigma$ be a flag simplicial complex and consider its Stanley-Reisner ring $\mathbb{F}[\Sigma]=\mathbb{F}\left[z_{i} \mid\{i\} \in \Sigma\right] / I_{\Sigma}$, which is a Koszul algebra. It was already known to Fröberg [Frö75] how to explicitly describe a minimal $\mathbb{F}[\Sigma]$-resolution of the residue field $\mathbb{F}$ : we will briefly describe such a generalized Koszul complex here, following the treatment in [MP15, Section 8].

The Koszul algebra $\mathbb{F}[\Sigma]$ admits a Koszul dual algebra $\mathbb{F}[\Sigma]$ !, which is obtained by quotienting the noncommutative polynomial ring $\mathbb{F}\left\langle Z_{i} \mid\{i\} \in \Sigma\right\rangle$ by the relations

$$
\begin{gathered}
Z_{i}^{2} \text { for every vertex } i \text { of } \Sigma \\
Z_{i} Z_{j}+Z_{j} Z_{i} \text { for every edge }\{i, j\} \text { of } \Sigma .
\end{gathered}
$$

In particular, given a noncommutative monomial in the $Z$-variables, two consecutive distinct variables $v$ and $v^{\prime}$ can anticommute unless $v v^{\prime} \in I_{\Sigma}$.

Notation 5.4. In what follows we will think of noncommutative monomials $\mathbf{w}$ in the $Z$ variables as words, and will denote by [w] the equivalence class of $\mathbf{w}$ with respect to the relations (5.1) (sometimes writing $[-\mathbf{w}]$ to denote $-[\mathbf{w}]$ ). We will write $\mathbf{w} \| v$ for the word obtained by adding the letter $v$ at the end of the word $\mathbf{w}$. Finally, for any nonzero [w], the support $\operatorname{supp}([\mathbf{w}])$ will be the signless commutative monomial obtained by multiplying together the $z$-variables corresponding to the letters of any representative of $[\mathbf{w}]$.

Definition 5.5. The generalized Koszul complex $(\mathbb{G} \mathbb{K} \cdot(\mathbb{F}[\Sigma]), \partial)$ is the chain complex of free $\mathbb{F}[\Sigma]$-modules given by the following data:

- $\mathbb{G}_{j}(\mathbb{F}[\Sigma])=\mathbb{F}[\Sigma] \otimes_{\mathbb{F}} \mathbb{F}[\Sigma]_{j}^{!}$;

- if $j>0$ and $\mathbf{w}=Z_{i_{1}} Z_{i_{2}} \ldots Z_{i_{j}}$ is a $j$-letter word, the differential $\partial$ is given by

$$
\partial(1 \otimes[\mathbf{w}])=\sum_{k \in \text { head }(\mathbf{w})}(-1)^{k-1} z_{i_{k}} \otimes\left[\mathbf{w} \backslash\left\{Z_{i_{k}}\right\}\right],
$$

where $\mathbf{w} \backslash\left\{Z_{i_{k}}\right\}$ is the $(j-1)$-letter word obtained by erasing the letter $Z_{i_{k}}$ from $\mathbf{w}$ and $\operatorname{head}(\mathbf{w})$ is the set of those indices $k$ for which there exists a representative of $[\mathbf{w}]$ with $Z_{i_{k}}$ as its first letter. One can check that such a differential is indeed well-defined.

Example 5.6. Let $\Sigma=\{12,23,1,2,3, \varnothing\}$.

Then an $\mathbb{F}$-basis for $\mathbb{F}[\Sigma]_{2}^{!}$is $\left\{\left[Z_{1} Z_{2}\right],\left[Z_{1} Z_{3}\right],\left[Z_{2} Z_{3}\right],\left[Z_{3} Z_{1}\right]\right\}$, whereas an $\mathbb{F}$-basis for $\mathbb{F}[\Sigma]_{3}^{!}$ is $\left\{\left[Z_{1} Z_{2} Z_{3}\right],\left[Z_{1} Z_{3} Z_{1}\right],\left[Z_{2} Z_{3} Z_{1}\right],\left[Z_{3} Z_{1} Z_{3}\right]\right\}$.

Note that, for instance, $\left[Z_{1}^{2}\right]=0$ and $\left[Z_{2} Z_{3} Z_{1}\right]=-\left[Z_{3} Z_{2} Z_{1}\right]=\left[Z_{3} Z_{1} Z_{2}\right]$, but $\left[Z_{2} Z_{3} Z_{1}\right] \neq$ $\left[Z_{2} Z_{1} Z_{3}\right]$. If $\mathbf{w}=Z_{2} Z_{3} Z_{1}$, we have that head $(\mathbf{w})=\{1,2\}$ and

$$
\partial\left(1 \otimes\left[Z_{2} Z_{3} Z_{1}\right]\right)=z_{2} \otimes\left[Z_{3} Z_{1}\right]-z_{3} \otimes\left[Z_{2} Z_{1}\right]=z_{2} \otimes\left[Z_{3} Z_{1}\right]+z_{3} \otimes\left[Z_{1} Z_{2}\right] .
$$

The matrix describing the map $\mathbb{G}_{3}(\mathbb{F}[\Sigma]) \stackrel{\partial}{\rightarrow} \mathbb{G}_{2}(\mathbb{F}[\Sigma])$ in the proposed basis is

$\begin{aligned} & 1 \otimes\left[Z_{1} Z_{2}\right] \\ & 1 \otimes\left[Z_{1} Z_{3}\right] \\ & 1 \otimes\left[Z_{2} Z_{3}\right] \\ & 1 \otimes\left[Z_{3} Z_{1}\right]\end{aligned}\left(\begin{array}{cccc}\left.Z_{2} Z_{3}\right] & 1 \otimes\left[Z_{1} Z_{3} Z_{1}\right] & 1 \otimes\left[Z_{2} Z_{3} Z_{1}\right] & 1 \otimes\left[Z_{3} Z_{1} Z_{3}\right] \\ 0 & 0 & z_{3} & 0 \\ -z_{2} & 0 & 0 & z_{3} \\ z_{1} & 0 & 0 & 0 \\ 0 & z_{1} & z_{2} & 0\end{array}\right)$.


5.2. Homological properties of Stanley-Reisner rings of flag Bier balls. For the rest of this section we fix a pure flag simplicial complex $\Delta$ and the associated Bier ball $\Gamma$. In the following we will be interested in $\mathbb{N}^{2 n}$-graded objects; we will reserve the word "multidegree" to denote either a vector in $\mathbb{N}^{2 n}$ or the associated monomial in $x_{1}, \ldots, x_{n}, y_{1}, \ldots, y_{n}$. When working with multidegrees, we shall distinguish between two types of variables.

Definition 5.7. Let $\mathbf{m}$ be a multidegree. A variable $v \mid \mathbf{m}$ is red with respect to $\mathbf{m}$ (and $\Gamma$ ) if there exists $v^{\prime} \mid \mathbf{m}$ such that $v v^{\prime} \in I_{\Gamma}$. Observe that necessarily $v \neq v^{\prime}$. A variable $v \mid \mathbf{m}$ which is not red is called blue. We say that a multidegree is blue if all the variables in its support are blue, and red otherwise.

Remark 5.8. By definition, blue multidegrees correspond bijectively to monomials of $\mathbb{F}[\Gamma]$. In particular, any $\mathbb{Z}^{2 n}$-graded ideal of $\mathbb{F}[\Gamma]$ is generated by a collection of blue monomials.

We now establish two technical lemmas about the homological behaviour of $\mathbb{Z}^{2 n}$-graded ideals in $\mathbb{F}[\mathbf{x}, \mathbf{y}]$ and $\mathbb{F}[\Gamma]$ : these results will be crucial for the proof of Theorem 5.1.

Lemma 5.9 (Blue Lemma). Let $\mathbf{m}$ be a blue multidegree, let $\mathcal{M}$ be a collection of blue monomials, and denote by $I^{\mathcal{M}}$ (respectively, $J^{\mathcal{M}}$ ) the $\mathbb{Z}^{2 n}$-graded ideal of $\mathbb{F}[\mathbf{x}, \mathbf{y}]$ (respectively, $\mathbb{F}[\Gamma])$ generated by $\mathcal{M}$. Then:

$i$. if $\mathbf{n}$ divides $\mathbf{m}$, then $\mathbf{n}$ is also a blue multidegree;

ii. the $\mathbb{F}$-vector spaces $I_{\mathbf{m}}^{\mathcal{M}}$ and $J_{\mathbf{m}}^{\mathcal{M}}$ are either both one-dimensional or both $\{0\}$;

iii. $\mathbb{G} \mathbb{K}_{\bullet}(\mathbb{F}[\Gamma])_{\mathbf{m}}=\mathbb{K}_{\bullet}(\mathbf{x}, \mathbf{y})_{\mathbf{m}}$, where $\mathbb{K}_{\bullet}(\mathbf{x}, \mathbf{y})$ is the usual Koszul complex on the variables $\mathbf{x}$ and $\mathbf{y}$;

iv. $\beta_{i, \mathbf{m}}^{\mathbb{F}[\Gamma]}\left(J^{\mathcal{M}}\right)=\beta_{i, \mathbf{m}}^{\mathbb{F}[\mathbf{x}, \mathbf{y}]}\left(I^{\mathcal{M}}\right)$ for every $i \in \mathbb{N}$.

Proof:

i. This is a direct consequence of the definition of blue multidegree.

ii. Both $J_{\mathbf{m}}^{\mathcal{M}}$ and $I_{\mathbf{m}}^{\mathcal{M}}$ can be at most one-dimensional; if they differ, then it must be that $\mathbf{m} \in I_{\Gamma}$, but this cannot happen precisely because $\mathbf{m}$ is blue.

iii. Fix $j \in \mathbb{N}$ and consider

$$
\mathbb{G} \mathbb{K}_{j}(\mathbb{F}[\Gamma])_{\mathbf{m}}=\left(\mathbb{F}[\Gamma] \otimes \mathbb{F}[\Gamma]_{j}^{!}\right)_{\mathbf{m}}=\bigoplus_{\substack{\mathbf{n}|\mathbf{m}\\| \mathbf{n} \mid=j}} \mathbb{F}[\Gamma]_{\frac{\mathbf{m}}{\mathbf{n}}} \otimes \mathbb{F}[\Gamma]_{\mathbf{n}}^{!}
$$

Pick a multidegree $\mathbf{n}$ dividing $\mathbf{m}$ and such that $|\mathbf{n}|=j$; by part $\mathrm{i}, \mathbf{n}$ is blue. For any word $\mathbf{w}$ with support $\mathbf{n}$, one has that $[\mathbf{w}] \neq 0$ if and only if $\mathbf{n}$ is squarefree: otherwise, since all letters can anticommute, we would be able to put next to each other two occurrences of the same letter, causing the whole class to vanish. We can hence assume that $\mathbf{n}$ is squarefree; in particular, since $x_{h} y_{h} \in I_{\Gamma}$ for every $1 \leq h \leq n$, we can write $\mathbf{n}$ as $z_{i_{1}} z_{i_{2}} \ldots z_{i_{j}}$, where $i_{1}<i_{2}<\ldots<i_{j}$ and each $z_{i_{k}}$ is either $x_{i_{k}}$ or $y_{i_{k}}$. All words $\mathbf{w}$ with support $\mathbf{n}$ belong to the same class (up to a global sign), since the order of the letters does not really matter in this case: we will denote such class by $z_{i_{1}} z_{i_{2}} \ldots z_{i_{j}}$. Such a characterization proves that $\mathbb{G}_{j}(\mathbb{F}[\Gamma])_{\mathbf{m}}$ coincides with $\mathbb{K}_{j}(\mathbf{x}, \mathbf{y})_{\mathbf{m}}$. As for the differential, since head $\left(z_{i_{1}} z_{i_{2}} \ldots z_{i_{j}}\right)=\{1,2, \ldots, j\}$, we get

$$
\partial\left(\frac{\mathbf{m}}{\mathbf{n}} \otimes \mathbf{1}_{z_{i_{1}} z_{i_{2}} \ldots z_{i_{j}}}\right)=\sum_{k=1}^{j}(-1)^{k-1} \frac{z_{i_{k}} \mathbf{m}}{\mathbf{n}} \otimes \mathbf{1}_{z_{i_{1}} z_{i_{2}} \ldots \hat{z}_{i_{k}} \ldots z_{i_{j}}}
$$


i.e. the differential of the (degree $\mathbf{m}$ part of the) usual Koszul complex on the variables $\mathbf{x}$ and $\mathbf{y}$.

iv. It is enough to show that the chain complexes of $\mathbb{F}$-vector spaces $\left(J^{\mathcal{M}} \otimes \mathbb{G} \mathbb{K} \text {. }(\mathbb{F}[\Gamma])\right)_{\mathbf{m}}$ and $\left(I^{\mathcal{M}} \otimes \mathbb{K}_{\bullet}(\mathbf{x}, \mathbf{y})\right)_{\mathbf{m}}$ coincide.

For every $j \in \mathbb{N}$ one has that

$$
\left(J^{\mathcal{M}} \otimes \mathbb{G}_{j}(\mathbb{F}[\Gamma])\right)_{\mathbf{m}}=\bigoplus_{\mathbf{n} \mid \mathbf{m}} J_{\frac{\mathbf{m}}{\mathbf{n}}}^{\mathcal{M}} \otimes\left(\mathbb{G} \mathbb{K}_{j}(\mathbb{F}[\Gamma])\right)_{\mathbf{n}} ;
$$

by part $\mathrm{i}$, both $\mathbf{n}$ and $\frac{\mathbf{m}}{\mathbf{n}}$ are blue multidegrees. By parts ii and iii, one then has that $J_{\frac{\mathbf{m}}{\mathbf{n}}}^{\mathcal{M}}=I_{\frac{\mathbf{m}}{\mathbf{n}}}^{\mathcal{M}}$ and $\mathbb{G}_{j}(\mathbb{F}[\Gamma])_{\mathbf{n}}=\mathbb{K}_{j}(\mathbf{x}, \mathbf{y})_{\mathbf{n}}$. Analyzing the differential maps as in the proof of part iii yields the claim.

Lemma 5.10 (Red Lemma). Let $J$ be a $\mathbb{Z}^{2 n}$-graded ideal of $\mathbb{F}[\Gamma]$, let $\mathbf{m}$ be a red multidegree and assume that $\beta_{i, \mathbf{m}}(J) \neq 0$ for some $i>0$. Then there exists a red variable $v$ such that $\beta_{i-1, \frac{\mathrm{m}}{v}}(J) \neq 0$.

Proof: Let us start by considering a cycle $z$ which has homological degree $i$, internal multidegree $\mathbf{m}$ and is not a boundary. Since $\operatorname{Tor}_{i}(J, \mathbb{F})_{\mathbf{m}}=H_{i}(J \otimes \mathbb{G} \mathbb{K} \cdot(\mathbb{F}[\Gamma]))_{\mathbf{m}}$, such a cycle can be written as

$$
z=\sum_{j \in \mathcal{C}} \lambda_{j} n_{j} \otimes\left[\mathbf{w}_{j}\right]
$$

where for each $j$ in the finite set $\mathcal{C}$ one has that $\lambda_{j} \in \mathbb{F} \backslash\{0\}, n_{j}$ is a monomial in $J$, [ $\mathbf{w}_{j}$ ] is the nonzero class of a word $\mathbf{w}_{j}$ with $i$ letters, and $n_{j} \cdot \operatorname{supp}\left(\left[\mathbf{w}_{j}\right]\right)=\mathbf{m}$. Note that each word $\mathbf{w}_{j}$ in (5.3) must contain at least one red letter $v$. If this were not the case for some $\mathbf{w}_{j}$, then any two red variables $v$ and $\bar{v}$ such that $v \bar{v} \in I_{\Gamma}$ would both divide $n_{j}$, causing the whole term to vanish.

Since blue letters anticommute with every other letter, there must be at least a red letter $v_{1}$ that appears as the last letter of a representative of some $\left[\mathbf{w}_{j}\right]$, up to sign. Let us fix such a $v_{1}$. By possibly tweaking the sign of some $\lambda_{j}$, we can assume without loss of generality that

$$
z=\sum_{j \in \mathcal{A}} \lambda_{j} n_{j} \otimes\left[\tilde{\mathbf{w}}_{j} \| v_{1}\right]+\sum_{j \in \mathcal{B}} \lambda_{j} n_{j} \otimes\left[\mathbf{w}_{j}\right]=: z_{v_{1}}+z^{\prime}
$$

where each $\tilde{\mathbf{w}}_{j}$ has $i-1$ letters, $\mathcal{A} \sqcup \mathcal{B}=\mathcal{C}$, and the nonempty set $\mathcal{A}$ contains all the indices for which $v_{1}$ can travel to the end of the associated word.

Claim: $z_{v_{1}}$ is a cycle (and hence so is $z^{\prime}$ ).

To see this, note that all the basis elements appearing in $\partial\left(1 \otimes\left[\tilde{\mathbf{w}}_{j} \| v_{1}\right]\right)$ must end in $v_{1}$, except possibly for those words $\tilde{\mathbf{w}}_{j} \| v_{1}$ where $v_{1}$ is free to navigate to the front. However, this last instance can happen only if the support of $\tilde{\mathbf{w}}_{j}$ contains no variable $\bar{v}_{1}$ with $v_{1} \bar{v}_{1} \in I_{\Gamma}$; then, since $\bar{v}_{1}$ divides $\mathbf{m}$, it must be that $\bar{v}_{1}$ divides $n_{j}$. But then, when $v_{1}$ exits the word, it multiplies $\bar{v}_{1}$ and vanishes. Hence, $\partial z_{v_{1}}$ can be written as a combination of words ending in $v_{1}$. On the other hand, $\partial z^{\prime}$ can contain no such words. But then, since $\partial z=0$, it must be that $\partial z_{v_{1}}=\partial z^{\prime}=0$. Note that both of these new cycles have again homological degree $i$ and internal multidegree $\mathbf{m}$. If $z_{v_{1}}$ is not a boundary, we keep it and we stop. If instead $z_{v_{1}}$ is a boundary, then $z^{\prime}$ cannot be so (otherwise the original $z$ would also be a boundary) and 
we can iterate the same procedure as before, finding a new red variable $v_{2} \neq v_{1}$ and writing $z^{\prime}=z_{v_{2}}^{\prime}+z^{\prime \prime}$. This process can be repeated only finitely many times and will yield the desired non-boundary cycle.

From now on we can hence assume without loss of generality that

$$
z=\sum_{j \in \mathcal{A}} \lambda_{j} n_{j} \otimes\left[\tilde{\mathbf{w}}_{j} \| v\right]
$$

Now let

$$
\hat{z}:=\sum_{j \in \mathcal{A}} \lambda_{j} n_{j} \otimes\left[\tilde{\mathbf{w}}_{j}\right]
$$

Reasoning as in the proof of the claim above, it follows that $\hat{z}$ is a cycle; moreover, if there existed $\hat{y}$ such that $\partial \hat{y}=\hat{z}$, then $z$ would also be a boundary (appending $v$ at the end of each word in $\hat{y}$ ). By definition, the cycle $\hat{z}$ has homological degree $i-1$ and internal multidegree $\frac{\mathrm{m}}{v}$, and this concludes the proof.

Corollary 5.11. Let $\mathcal{M}$ be a collection of blue monomials, and denote by $I^{\mathcal{M}}$ (respectively, $J^{\mathcal{M}}$ ) the $\mathbb{Z}^{2 n}$-graded ideal of $\mathbb{F}[\mathbf{x}, \mathbf{y}]$ (respectively, $\mathbb{F}[\Gamma]$ ) generated by $\mathcal{M}$. Let $k$ and $h$ be two nonnegative integers. Then:

i. for every $i, j$ for which $\beta_{i, j}^{\mathbb{F}[\Gamma]}\left(J^{\mathcal{M}}\right) \neq 0$ there exists $0 \leq h \leq i$ for which $\beta_{i-h, j-h}^{\mathbb{F}[\mathbf{x}, \mathbf{y}]}\left(I^{\mathcal{M}}\right) \neq 0$;

ii. if $\beta_{i, j}^{\mathbb{F}[\mathbf{x}, \mathbf{y}]}\left(I^{\mathcal{M}}\right)=0$ for every $0 \leq i \leq k$ and $j>i+h$, then $\beta_{i, j}^{\mathbb{F}[\Gamma]}\left(J^{\mathcal{M}}\right)=0$ for every $0 \leq i \leq k$ and $j>i+h$; in particular, if the $\mathbb{F}[\mathbf{x}, \mathbf{y}]$-resolution of $I^{\mathcal{M}}$ is linear for $k$ steps, so is the $\mathbb{F}[\Gamma]$-resolution of $J^{\mathcal{M}}$;

iii. $\operatorname{reg}_{\mathbb{F}[\Gamma]}\left(J^{\mathcal{M}}\right) \leq \operatorname{reg}_{\mathbb{F}[\mathbf{x}, \mathbf{y}]}\left(I^{\mathcal{M}}\right)$.

If moreover all the monomials in $\mathcal{M}$ contain only y-variables, then:

iv. if $\beta_{i, j}^{\mathbb{F}[\mathbf{x}, \mathbf{y}]}\left(I^{\mathcal{M}}\right) \neq 0$, then $\beta_{i, j}^{\mathbb{F}[\Gamma]}\left(J^{\mathcal{M}}\right) \neq 0$;

v. $\beta_{i, j}^{\mathbb{F}[\mathbf{x}, \mathbf{y}]}\left(I^{\mathcal{M}}\right)=0$ for every $0 \leq i \leq k$ and $j>i+h$ if and only if $\beta_{i, j}^{\mathbb{F}[\Gamma]}\left(J^{\mathcal{M}}\right)=0$ for every $0 \leq i \leq k$ and $j>i+h$;

vi. $\operatorname{reg}_{\mathbb{F}[\Gamma]}\left(J^{\mathcal{M}}\right)=\operatorname{reg}_{\mathbb{F}[\mathbf{x}, \mathbf{y}]}\left(I^{\mathcal{M}}\right)$. In particular, $J^{\mathcal{M}}$ has a linear $\mathbb{F}[\Gamma]$-resolution if and only if $I^{\mathcal{M}}$ has a linear $\mathbb{F}[\mathbf{x}, \mathbf{y}]$-resolution.

Proof:

i. Pick $i$ and $j$ such that $\beta_{i, j}^{\mathbb{F}[\Gamma]}\left(J^{\mathcal{M}}\right) \neq 0$, and choose a multidegree $\mathbf{m}$ such that $|\mathbf{m}|=j$ and $\beta_{i, \mathbf{m}}^{\mathbb{F}[\Gamma]}\left(J^{\mathcal{M}}\right) \neq 0$. If $\mathbf{m}$ is blue, Lemma 5.9.iv yields the claim with $h=0$. If instead $\mathbf{m}$ is red, we know by Lemma 5.10 that we can decrease the homological index from $i$ to $i-1$ while staying in the $(j-i)$-th linear strand of the $\mathbb{F}[\Gamma]$-resolution of $J^{\mathcal{M}}$. This can be repeated until we hit a blue multidegree, which happens at the latest when we reach the zeroth homological degree, since all generators of $J^{\mathcal{M}}$ are blue. Then applying Lemma 5.9.iv finishes the proof.

ii.-iii. These statements follow from part i.

iv. Pick $i$ and $j$ such that $\beta_{i, j}^{\mathbb{F}[\mathbf{x}, \mathbf{y}]}\left(I^{\mathcal{M}}\right) \neq 0$, and choose a multidegree $\mathbf{m}$ such that $|\mathbf{m}|=j$ and $\beta_{i, \mathbf{m}}^{\mathbb{F}[\mathbf{x}, \mathbf{y}]}\left(I^{\mathcal{M}}\right) \neq 0$. Since no $x$-variables are involved in any minimal generator of $I^{\mathcal{M}}$, one has that $\beta_{i, \mathbf{m}}^{\mathbb{F}[\mathbf{x}, \mathbf{y}}\left(I^{\mathcal{M}}\right)$ can be nonzero only if $\mathbf{m}$ is a monomial in the $y$ variables; however, any such $\mathbf{m}$ must be blue, as by construction the only variable 
$v$ with $v y_{i} \in I_{\Gamma}$ is $v=x_{i}$. Applying Lemma 5.9.iv then yields that $\beta_{i, \mathbf{m}}^{\mathbb{F}[\Gamma]}\left(J^{\mathcal{M}}\right)=$ $\beta_{i, \mathbf{m}}^{\mathbb{F}[\mathbf{x}, \mathbf{y}]}\left(I^{\mathcal{M}}\right)$, and the claim follows.

v.--vi. These statements follow from combining parts $i$ and iv.

Remark 5.12. Under the hypotheses of Corollary 5.11.iv-vi, the Betti table of $J^{\mathcal{M}}$ is a "horizontal echo" of the (finite) Betti table of $I^{\mathcal{M}}$.

As an example, take $\mathbb{F}[\Gamma]=\mathbb{F}[\mathbf{x}, \mathbf{y}] /\left(x_{1} x_{2}, x_{1} x_{3}, x_{1} y_{1}, x_{2} y_{2}, x_{3} y_{3}\right)$ and $\mathcal{M}=\left\{y_{1} y_{2}, y_{2}^{2} y_{3}^{2}, y_{3}^{4}\right\}$. Using Macaulay2 [GS21], we find out that the Betti tables of $I^{\mathcal{M}}$ and $J^{\mathcal{M}}$ look as follows:

\begin{tabular}{l|lll} 
& 0 & 1 & 2 \\
\hline 2 & $\mathbf{1}$ & $\cdot$ & $\cdot$ \\
3 & $\cdot$ & $\cdot$ & $\cdot$ \\
4 & $\mathbf{2}$ & $\mathbf{1}$ &. \\
5 &. & $\mathbf{2}$ & $\mathbf{1}$
\end{tabular}

\begin{tabular}{c|cccccccc} 
& 0 & 1 & 2 & 3 & 4 & 5 & 6 & 7 \\
\hline 2 & $\mathbf{1}$ & 2 & 5 & 13 & 34 & 89 & 233 & 610 \\
3 &. &. &. &. &. &. &. &. \\
4 & $\mathbf{2}$ & $\mathbf{4}$ & 10 & 26 & 68 & 178 & 466 & 1220 \\
5 &. & $\mathbf{2}$ & $\mathbf{6}$ & 16 & 42 & 110 & 288 & 754
\end{tabular}

TABLE 1. The Betti table of $I^{\mathcal{M}}$ (left) and the beginning of the Betti table of $J^{\mathcal{M}}$ (right) from Remark 5.12.

Remark 5.13. Note that the statements of Corollary 5.11.iv-vi can indeed fail when the elements of $\mathcal{M}$ are not in the $y$-variables only.

For instance, take $\mathbb{F}[\Gamma]=\mathbb{F}[\mathbf{x}, \mathbf{y}] /\left(x_{1} x_{3}, x_{1} y_{1}, x_{2} y_{2}, x_{3} y_{3}, x_{4} y_{4}\right)$ and $\mathcal{M}=\left\{x_{1} x_{2}, x_{3} x_{4}\right\}$. Since $I^{\mathcal{M}}=\left(x_{1} x_{2}, x_{3} x_{4}\right)$ is a complete intersection in $\mathbb{F}[\mathbf{x}, \mathbf{y}]$, one has that $\operatorname{reg}_{\mathbb{F}[\mathbf{x}, \mathbf{y}]}\left(I^{\mathcal{M}}\right)=3$. However, since $J^{\mathcal{M}}$ has linear quotients with respect to the Koszul filtration consisting of all subsets of variables of $\mathbb{F}[\Gamma]$, then $J^{\mathcal{M}}$ has a 2-linear $\mathbb{F}[\Gamma]$-resolution [CDNR13, Lemma 17]; hence, $2=\operatorname{reg}_{\mathbb{F}[\Gamma]}\left(J^{\mathcal{M}}\right)<\operatorname{reg}_{\mathbb{F}[\mathbf{x}, \mathbf{y}]}\left(I^{\mathcal{M}}\right)=3$.

We are finally ready to prove Theorem 5.1.

Proof: (of Theorem 5.1) Let $\mathcal{M}:=\left\{\mathbf{y}^{[n] \backslash F} \mid F\right.$ facet of $\left.\Delta\right\}$, and let $I^{\mathcal{M}} \subseteq \mathbb{F}[\mathbf{x}, \mathbf{y}], J^{\mathcal{M}} \subseteq \mathbb{F}[\Gamma]$ be as in Corollary 5.11. Note that $I^{\mathcal{M}}$ is the extension of the Alexander dual ideal of $\Delta$ to a polynomial ring containing also $x$-variables. By Theorem 2.6, the simplicial complex $\Delta$ is $\left(S_{k}\right)$ if and only if the Alexander dual ideal of $\Delta$ has an $\mathbb{F}[\mathbf{y}]$-resolution which is linear for $k-1$ steps. This is equivalent to stating that $I^{\mathcal{M}}$ has an $\mathbb{F}[\mathbf{x}, \mathbf{y}]$-resolution which is linear for $k-1$ steps. By Corollary 5.11.v, this is in turn equivalent to stating that $J^{\mathcal{M}}$ has an $\mathbb{F}[\Gamma]$-resolution which is linear for $k-1$ steps. By Proposition 3.4.iv, the ideal $J^{\mathcal{M}}$ coincides with the canonical module $\omega_{\mathbb{F}[\Gamma]}$. Finally, applying Lemma 2.13 with $A=\mathbb{F}[\Gamma]$, $M=\omega_{\mathbb{F}[\Gamma]}(-a(\mathbb{F}[\Gamma])-1)$ concludes the proof.

Remark 5.14. In [MSS21a] the authors consider the problem of characterizing the pairs of positive integers $(c, r)$ for which there exists a non-Koszul quadratic Gorenstein algebra $R$ with codimension $c$ and regularity $r$. The results of [MSS21a], [MSS21b] and [MS20] leave only two cases open, namely $(c, r)=(6,3)$ and $(c, r)=(7,3)$. One might wonder if the results in this section provide examples of non-Koszul quadratic Gorenstein algebras with 
such invariants. However, this is not the case: if $R_{\Delta}$ has regularity 3 , then $\Delta$ must be 1 dimensional, and in this case $\Delta$ satisfies $\left(S_{2}\right)$ if and only if it is Cohen-Macaulay (over any field).

5.3. An application: quadratic Gorenstein algebras which are not Koszul in prescribed characteristics. We conclude this section with an application of Corollary 5.3. Given a finite list of prime numbers $P=\left\{p_{1}, \ldots, p_{m}\right\}$, our result can be used to construct quadratic Gorenstein $\mathbb{F}$-algebras which are Koszul if and only if $\operatorname{char}(\mathbb{F}) \notin P$. Moreover, the presentations of these algebras will be characteristic-free, see Proposition 4.3.

To do so, it suffices to exhibit a flag simplicial complex which is Cohen-Macaulay over $\mathbb{F}$ if and only if the characteristic of $\mathbb{F}$ is not in $P$. Note that such a complex will automatically be $\left(S_{2}\right)$ (which corresponds to $R_{\Delta}$ having a quadratic presentation, see Proposition 4.4), since the $\left(S_{2}\right)$ property is characteristic-free and the complex is Cohen-Macaulay in some characteristic.

We will focus on flag triangulations of 3-dimensional lens spaces. A 3-dimensional lens space is an orientable 3-manifold obtained as a quotient of the 3-sphere by certain rotations (see [Hat02, Example 2.43]). Such spaces are parametrized by two coprime integers $p, q \geq 1$, and $L\left(p_{1}, q_{1}\right) \cong L\left(p_{2}, q_{2}\right)$ if and only if $p_{1}=p_{2}$ and $q_{1}= \pm q_{1}^{ \pm 1} \bmod p$. The reduced integral homology groups of $L(p, q)$ are as follows: $\tilde{H}_{0}(L(p, q))=\tilde{H}_{2}(L(p, q))=0, \tilde{H}_{3}(L(p, q))=\mathbb{Z}$ and $\tilde{H}_{1}(L(p, q))=\mathbb{Z}_{p}$. As lens spaces are homology 3-manifolds, the link of any nonempty face in any triangulation $\Delta$ of $L(p, q)$ is a homology sphere. Hence, by Reisner's criterion together with the universal coefficient theorem [Hat02, Section 3A], $\Delta$ is Cohen-Macaulay over a field $\mathbb{F}$ if and only if $\tilde{H}_{1}(\Delta ; \mathbb{F})=\tilde{H}_{1}(\Delta ; \mathbb{Z}) \otimes \mathbb{F}=\mathbb{F} / p \mathbb{F}=0$. In particular, every triangulation of $L(p, q)$ is Cohen-Macaulay over $\mathbb{F}$ if and only if $\operatorname{char}(\mathbb{F}) \neq p$. Therefore if $\Delta$ is a flag triangulation of $L(p, q)$, then $R_{\Delta}$ is a quadratic Gorenstein $\mathbb{F}$-algebra which is Koszul if and only if $\operatorname{char}(\mathbb{F}) \neq p$. We will generalize this fact by considering connected sums of lens spaces.

Proposition 5.15. Let $P=\left\{p_{1}, \ldots, p_{m}\right\}$ be a set of prime numbers. Let $\Delta$ be any flag triangulation of the connected sum $L\left(p_{1}, q_{1}\right) \# \cdots \# L\left(p_{m}, q_{m}\right)$, for some positive integers $q_{1}, \ldots, q_{m}$. Then $R_{\Delta}$ is a quadratic Gorenstein $\mathbb{F}$-algebra that is Koszul if and only if char $(\mathbb{F}) \notin P$.

Proof: $\quad$ As $\Delta$ is a triangulated 3-manifold, one has that $\tilde{H}_{i}\left(\operatorname{lk}_{\Delta}(F) ; \mathbb{Z}\right)=0$ for every $\varnothing \neq F \in \Delta$ and for every $i<\operatorname{dim}(F)$. Moreover, we can compute the homology of a connected sum of manifolds by a standard application of the Mayer-Vietoris sequence. This yields that $\tilde{H}_{2}(\Delta ; \mathbb{Z})=0$ and $\tilde{H}_{1}(\Delta ; \mathbb{Z})=\bigoplus_{p \in P} \mathbb{Z} / p \mathbb{Z}$. Thus, we have that $\Delta$ is Cohen-Macaulay over a field $\mathbb{F}$ if and only if $\tilde{H}_{1}(\Delta ; \mathbb{Z}) \otimes \mathbb{F}=\bigoplus_{p \in P} \mathbb{F} / p \mathbb{F}=0$, which in turn happens if and only if $\operatorname{char}(\mathbb{F}) \notin P$. We conclude by using Corollary 5.3. $\square$ Requiring that $\Delta$ is flag is

not restrictive, as any triangulable space has a flag triangulation given, for example, by its barycentric subdivision [Sta96, III.4]. However, these simplicial complexes typically have a lot of vertices, and it is a challenging problem to find vertex-minimal flag triangulations of a given space.

Finally, we observe that Proposition 5.15 can be applied verbatim to flag triangulations of higher-dimensional lens spaces, yielding quadratic Gorenstein algebras with the same behaviour as in Proposition 5.15 but with an $h$-polynomial of higher degree. 
Example 5.16. Let $\Delta$ be a flag triangulation of $\mathbb{R P}^{2}$. In $\left[\mathrm{BOW}^{+} 20\right]$ the authors construct two such non-isomorphic triangulations with the same $f$-vector $f(\Delta)=(1,11,30,20)$. We let $\Delta$ be the simplicial complex whose list of facets is reported in the left column of $\left[\mathrm{BOW}^{+} 20\right.$, Table 1]. The algebra $R_{\Delta}$ is then presented as $T / J$, with

$$
\begin{aligned}
T=\mathbb{F}[ & x_{1}, \ldots, x_{9}, x_{a}, x_{b}, y_{1}, \ldots, y_{9}, y_{a}, y_{b}, z_{145}, z_{126}, z_{156}, z_{237}, z_{347}, z_{267}, \\
& \left.z_{148}, z_{478}, z_{129}, z_{189}, z_{23 a}, z_{34 a}, z_{45 a}, z_{29 a}, z_{56 b}, z_{67 b}, z_{78 b}, z_{89 b}, z_{5 a b}, z_{9 a b}\right]
\end{aligned}
$$

and

$$
\begin{aligned}
& J=\left(x_{b} z_{145}, x_{a} z_{145}, x_{9} z_{145}, x_{8} z_{145}, x_{7} z_{145}, x_{6} z_{145}, x_{3} z_{145}, x_{2} z_{145}, x_{b} z_{126}, x_{a} z_{126}, x_{9} z_{126}, x_{8} z_{126},\right. \\
& x_{7} z_{126}, x_{5} z_{126}, x_{4} z_{126}, x_{3} z_{126}, x_{b} z_{156}, x_{a} z_{156}, x_{9} z_{156}, x_{8} z_{156}, x_{7} z_{156}, x_{4} z_{156}, x_{3} z_{156}, x_{2} z_{156}, \\
& x_{b} z_{237}, x_{a} z_{237}, x_{9} z_{237}, x_{8} z_{237}, x_{6} z_{237}, x_{5} z_{237}, x_{4} z_{237}, x_{1} z_{237}, x_{b} z_{347}, x_{a} z_{347}, x_{9} z_{347}, x_{8} z_{347}, \\
& x_{6} z_{347}, x_{5} z_{347}, x_{2} z_{347}, x_{1} z_{347}, x_{b} z_{267}, x_{a} z_{267}, x_{9} z_{267}, x_{8} z_{267}, x_{5} z_{267}, x_{4} z_{267}, x_{3} z_{267}, x_{1} z_{267} \text {, } \\
& x_{b} z_{148}, x_{a} z_{148}, x_{9} z_{148}, x_{7} z_{148}, x_{6} z_{148}, x_{5} z_{148}, x_{3} z_{148}, x_{2} z_{148}, x_{b} z_{478}, x_{a} z_{478}, x_{9} z_{478}, x_{6} z_{478} \text {, } \\
& x_{5} z_{478}, x_{3} z_{478}, x_{2} z_{478}, x_{1} z_{478}, x_{b} z_{129}, x_{a} z_{129}, x_{8} z_{129}, x_{7} z_{129}, x_{6} z_{129}, x_{5} z_{129}, x_{4} z_{129}, x_{3} z_{129} \text {, } \\
& x_{b} z_{189}, x_{a} z_{189}, x_{7} z_{189}, x_{6} z_{189}, x_{5} z_{189}, x_{4} z_{189}, x_{3} z_{189}, x_{2} z_{189}, x_{b} z_{23 a}, x_{9} z_{23 a}, x_{8} z_{23 a}, x_{7} z_{23 a}, \\
& x_{6} z_{23 a}, x_{5} z_{23 a}, x_{4} z_{23 a}, x_{1} z_{23 a}, x_{b} z_{34 a}, x_{9} z_{34 a}, x_{8} z_{34 a}, x_{7} z_{34 a}, x_{6} z_{34 a}, x_{5} z_{34 a}, x_{2} z_{34 a}, x_{1} z_{34 a}, \\
& x_{b} z_{45 a}, x_{9} z_{45 a}, x_{8} z_{45 a}, x_{7} z_{45 a}, x_{6} z_{45 a}, x_{3} z_{45 a}, x_{2} z_{45 a}, x_{1} z_{45 a}, x_{b} z_{29 a}, x_{8} z_{29 a}, x_{7} z_{29 a}, x_{6} z_{29 a}, \\
& x_{5} z_{29 a}, x_{4} z_{29 a}, x_{3} z_{29 a}, x_{1} z_{29 a}, x_{a} z_{56 b}, x_{9} z_{56 b}, x_{8} z_{56 b}, x_{7} z_{56 b}, x_{4} z_{56 b}, x_{3} z_{56 b}, x_{2} z_{56 b}, x_{1} z_{56 b} \text {, } \\
& x_{a} z_{67 b}, x_{9} z_{67 b}, x_{8} z_{67 b}, x_{5} z_{67 b}, x_{4} z_{67 b}, x_{3} z_{67 b}, x_{2} z_{67 b}, x_{1} z_{67 b}, x_{a} z_{78 b}, x_{9} z_{78 b}, x_{6} z_{78 b}, x_{5} z_{78 b} \text {, } \\
& x_{4} z_{78 b}, x_{3} z_{78 b}, x_{2} z_{78 b}, x_{1} z_{78 b}, x_{a} z_{89 b}, x_{7} z_{89 b}, x_{6} z_{89 b}, x_{5} z_{89 b}, x_{4} z_{89 b}, x_{3} z_{89 b}, x_{2} z_{89 b}, x_{1} z_{89 b} \text {, } \\
& x_{9} z_{5 a b}, x_{8} z_{5 a b}, x_{7} z_{5 a b}, x_{6} z_{5 a b}, x_{4} z_{5 a b}, x_{3} z_{5 a b}, x_{2} z_{5 a b}, x_{1} z_{5 a b}, x_{8} z_{9 a b}, x_{7} z_{9 a b}, x_{6} z_{9 a b}, x_{5} z_{9 a b}, \\
& \left.x_{4} z_{9 a b}, x_{3} z_{9 a b}, x_{2} z_{9 a b}, x_{1} z_{9 a b}\right) \\
& +\left(y_{4} z_{145}-y_{6} z_{156}, y_{2} z_{126}-y_{5} z_{156}, y_{2} z_{237}-y_{4} z_{347}, y_{1} z_{126}-y_{7} z_{267}, y_{3} z_{237}-y_{6} z_{267}, y_{5} z_{145}-y_{8} z_{148}\right. \text {, } \\
& y_{3} z_{347}-y_{8} z_{478}, y_{1} z_{148}-y_{7} z_{478}, y_{6} z_{126}-y_{9} z_{129}, y_{4} z_{148}-y_{9} z_{189}, y_{2} z_{129}-y_{8} z_{189}, y_{a} z_{23 a}-y_{7} z_{237} \text {, } \\
& y_{a} z_{34 a}-y_{7} z_{347}, y_{2} z_{23 a}-y_{4} z_{34 a}, y_{1} z_{145}-y_{a} z_{45 a}, y_{3} z_{34 a}-y_{5} z_{45 a}, y_{1} z_{129}-y_{a} z_{29 a}, y_{3} z_{23 a}-y_{9} z_{29 a} \text {, } \\
& y_{1} z_{156}-y_{b} z_{56 b}, y_{2} z_{267}-y_{b} z_{67 b}, y_{5} z_{56 b}-y_{7} z_{67 b}, y_{4} z_{45 a}-y_{b} z_{5 a b}, y_{a} z_{5 a b}-y_{6} z_{56 b}, y_{4} z_{478}-y_{b} z_{78 b} \text {, } \\
& \left.y_{6} z_{67 b}-y_{8} z_{78 b}, y_{1} z_{189}-y_{b} z_{89 b}, y_{7} z_{78 b}-y_{9} z_{89 b}, y_{2} z_{29 a}-y_{b} z_{9 a b}, y_{8} z_{89 b}-y_{a} z_{9 a b}, y_{5} z_{5 a b}-y_{9} z_{9 a b}\right) \\
& +\left(x_{2} x_{8}, x_{4} x_{6}, x_{3} x_{9}, x_{3} x_{6}, x_{7} x_{9}, x_{3} x_{8}, x_{6} x_{8}, x_{b} x_{2}, x_{4} x_{9}, x_{1} x_{a}, x_{5} x_{9}, x_{5} x_{7}, x_{6} x_{9}, x_{1} x_{7}, x_{a} x_{7},\right. \\
& \left.x_{1} x_{b}, x_{a} x_{6}, x_{2} x_{5}, x_{a} x_{8}, x_{2} x_{4}, x_{b} x_{4}, x_{3} x_{5}, x_{5} x_{8}, x_{b} x_{3}, x_{1} x_{3}\right) \\
& +\left(x_{1} y_{1}, x_{2} y_{2}, x_{3} y_{3}, x_{4} y_{4}, x_{5} y_{5}, x_{6} y_{6}, x_{7} y_{7}, x_{8} y_{8}, x_{9} y_{9}, x_{a} y_{a}, x_{b} y_{b}\right) \\
& +\left(z_{145}, z_{126}, z_{156}, z_{237}, z_{347}, z_{267}, z_{148}, z_{478}, z_{129}, z_{189}, z_{23 a}, z_{34 a}, z_{45 a}, z_{29 a}, z_{56 b}, z_{67 b}, z_{78 b},\right. \\
& \left.z_{89 b}, z_{5 a b}, z_{9 a b}\right)^{2} \text {. }
\end{aligned}
$$

The Hilbert series of the Gorenstein algebra $R_{\Delta}$ is equal to

$$
\frac{1+31 t+60 t^{2}+31 t^{3}+t^{4}}{(1-t)^{11}}
$$

We can verify Corollary 5.3 on this example. As any triangulation of the projective plane is Cohen-Macaulay over $\mathbb{F}$ if and only if the characteristic of the field is not equal to 2, we 
expect to observe a different behaviour for the resolution of $\mathbb{F}$ as an $R_{\Delta}$-module in the cases $\mathbb{F}=\mathbb{Z}_{2}$ and $\mathbb{F}=\mathbb{Z}_{3}$. Indeed, when $\mathbb{F}=\mathbb{Z}_{2}$, we find a nonlinear syzygy in homological degree 3 , as shown in Table 2. The numbers in Table 2 have been computed via Macaulay2 [GS21], using the commands use $(\mathrm{T} / \mathrm{J})$; betti res(ideal gens $(\mathrm{T} / \mathrm{J})$, LengthLimit $=>3$ ).

\begin{tabular}{c|cccc} 
& 0 & 1 & 2 & 3 \\
\hline 0 & 1 & 42 & 1297 & 37883 \\
1 &. &. &. & $\mathbf{1}$
\end{tabular}

\begin{tabular}{c|cccc} 
& 0 & 1 & 2 & 3 \\
\hline 0 & 1 & 42 & 1297 & 37883 \\
1 &. &. &. &.
\end{tabular}

TABLE 2. The beginning of the Betti tables of $\mathbb{F}$ as an $R_{\Delta}$-module in the cases $\mathbb{F}=\mathbb{Z}_{2}$ (left) and $\mathbb{F}=\mathbb{Z}_{3}$ (right). Here $\Delta$ is the flag triangulation of $\mathbb{R P}^{2}$ from Example 5.16.

\section{Quadratic Gröbner BASES AND SHELling ORDERS}

In this section we add a further connection between the combinatorial features of $\Delta$ and the algebraic properties of $R_{\Delta}$. We begin by recalling the notion of shellability.

Definition 6.1. A pure $(d-1)$-dimensional simplicial complex $\Delta$ is shellable if there exists a linear order $F_{1}, \ldots, F_{|\mathcal{F}(\Delta)|}$ of its facets such that $\Delta_{i-1} \cap\left\langle F_{i}\right\rangle$ is pure and $(d-2)$-dimensional for every $i=2, \ldots,|\mathcal{F}(\Delta)|$, where $\Delta_{i}=\left\langle F_{1}, \ldots, F_{i}\right\rangle$. The ordering $F_{1}, \ldots, F_{|\mathcal{F}(\Delta)|}$ is called a shelling order, and we will often refer to the step $\Delta_{i-1} \rightarrow \Delta_{i}$ as a shelling.

This property poses severe restrictions on the topology of $\Delta$, as shellable simplicial complexes are homotopy equivalent to a wedge of some (possibly zero) $(d-1)$-spheres. Boundary complexes of simplicial polytopes of any dimension are shellable, while there exist nonshellable simplicial spheres and balls already in dimension 3. Moreover, all links of a shellable simplicial complex are again shellable. Shellability has also implications on a more algebraic level, like the following.

Proposition 6.2. Shellable simplicial complexes are Cohen-Macaulay over any field.

The converse is far from being true and fails already in dimension 2: it is well known that any triangulation of the so-called dunce hat is Cohen-Macaulay over any field, but not shellable. However, the set of $f$-vectors of Cohen-Macaulay complexes and that of shellable ones coincide in any dimension [Sta77, Theorem 6].

The goal of the previous sections was to show a connection between the Cohen-Macaulayness of a flag simplicial complex $\Delta$ and the Koszulness of $R_{\Delta}$. The next result shows that we can tighten both conditions and still obtain a correspondence.

Theorem 6.3. Let $\Delta$ be a pure flag simplicial complex. Then $\Delta$ is shellable if and only if $R_{\Delta}$ has a quadratic Gröbner basis.

Recall that if a standard graded algebra has quadratic Gröbner basis, then it is Koszul. Before embarking on the proof of Theorem 6.3 we observe the following fact. 
Lemma 6.4. Any term order $<$ on $\mathbb{F}[\mathbf{x}, \mathbf{y}, \mathbf{z}]$ induces a total order $<$ on the facets of $\Delta$, defined as the reflexive closure of the following rule:

$$
F_{\ell}<F_{k} \Leftrightarrow \operatorname{in}_{<}\left(b_{F_{k}, F_{\ell}}\right)=\mathbf{y}^{F_{k} \backslash F_{\ell}} z_{F_{k}}
$$

Proof: $\quad$ By definition, for any two distinct facets $F_{k}$ and $F_{\ell}$ of $\Delta$ at most one of $F_{\ell}<F_{k}$ and $F_{k}<F_{\ell}$ holds. To conclude that $<$ is a partial order, we need to prove transitivity.

Let $F_{j}, F_{k}, F_{\ell}$ be facets such that $F_{j}>F_{k}, F_{k}>F_{\ell}$. We claim that $\operatorname{in}_{<}\left(b_{F_{j}, F_{\ell}}\right)=\mathbf{y}^{F_{j} \backslash F_{\ell}} z_{F_{j}}$. Indeed, multiplying $b_{F_{j}, F_{k}}$ by $\mathbf{y}^{F_{j} \cap F_{k}}$ yields that $\mathbf{y}^{F_{j}} z_{F_{j}}>\mathbf{y}^{F_{k}} z_{F_{k}}$; analogously, one has that $\mathbf{y}^{F_{k}} z_{F_{k}}>\mathbf{y}^{F_{\ell}} z_{F_{\ell}}$, and hence $\mathbf{y}^{F_{j}} z_{F_{j}}>\mathbf{y}^{F_{k}} z_{F_{k}}>\mathbf{y}^{F_{\ell}} z_{F_{\ell}}$. Since $\mathbf{y}^{F_{j}} z_{F_{j}}-\mathbf{y}^{F_{\ell}} z_{F_{\ell}}=\mathbf{y}^{F_{j} \cap F_{\ell}} b_{F_{j}, F_{\ell}}$, the claim follows.

Definition 6.5. If $<$ is a term order on $\mathbb{F}[\mathbf{x}, \mathbf{y}, \mathbf{z}]$, we will denote by $<$ be the unique total order on $\mathcal{F}(\Delta)$ induced by $<$ as in Lemma 6.4. Conversely, if $<$ is any total order on $\mathcal{F}(\Delta)$, we will say that a term order $<$ on $\mathbb{F}[\mathbf{x}, \mathbf{y}, \mathbf{z}]$ is compatible with $<$ if in $\operatorname{in}_{<}\left(b_{F_{k}, F_{\ell}}\right)=\mathbf{y}^{F_{k} \backslash F_{\ell}} z_{F_{k}}$ whenever $F_{\ell}<F_{k}$.

Lemma 6.6. Fix a term order $<$ on $\mathbb{F}[\mathbf{x}, \mathbf{y}, \mathbf{z}]$, let $<$ be the associated total order on $\mathcal{F}(\Delta)$ and let $F_{1}$ and $F_{2}$ be facets of $\Delta$ with $F_{1}>F_{2}$. Then the binomial $b_{F_{1}, F_{2}}$ can be reduced via the binomial $b_{G_{1}, G_{2}}$ if and only if $\left\{G_{1}, G_{2}\right\}=\left\{F_{1}, H\right\}$ for some facet $H$ such that $H<F_{1}$ and $F_{1} \cap F_{2} \subseteq H$. If this is the case, the binomial $b_{F_{1}, F_{2}}$ reduces to $\mathbf{y}^{F_{2} \cap\left(H \backslash F_{1}\right)} b_{H, F_{2}}$.

Proof: $\quad$ Since $F_{1}>F_{2}$, the leading term of $b_{F_{1}, F_{2}}$ is $\mathbf{y}^{F_{1} \backslash F_{2}} z_{F_{1}}$. We can operate a reduction via $b_{G_{1}, G_{2}}$ precisely when the leading term of $b_{G_{1}, G_{2}}$ divides $\mathbf{y}^{F_{1} \backslash F_{2}} z_{F_{1}}$. In order for this to happen, $b_{G_{1}, G_{2}}$ must be (up to sign) $b_{F_{1}, H}$, where $H$ is a facet of $\Delta$. For $z_{F_{1}}$ to appear in the leading term of $b_{F_{1}, H}$, it must be that $F_{1}>H$. Moreover, we need $\mathbf{y}^{F_{1} \backslash H}$ to divide $\mathbf{y}^{F_{1} \backslash F_{2}}$, i.e. $F_{1} \backslash H \subseteq F_{1} \backslash F_{2}$, which happens precisely when $F_{1} \cap F_{2} \subseteq H$ (note that this in turn equivalent to $F_{2} \backslash H \subseteq F_{2} \backslash F_{1}$ ). One checks that all these necessary conditions are indeed sufficient to get the desired reduction. When such conditions are met, one has that

$$
\begin{aligned}
b_{F_{1}, F_{2}} & =\mathbf{y}^{F_{1} \backslash F_{2}} z_{F_{1}}-\mathbf{y}^{F_{2} \backslash F_{1}} z_{F_{2}} \\
& =\mathbf{y}^{F_{1} \cap\left(H \backslash F_{2}\right)} \mathbf{y}^{F_{1} \backslash H} z_{F_{1}}-\mathbf{y}^{F_{2} \cap\left(H \backslash F_{1}\right)} \mathbf{y}^{F_{2} \backslash H} z_{F_{2}} \\
& =\mathbf{y}^{F_{1} \cap\left(H \backslash F_{2}\right)}\left(\mathbf{y}^{F_{1} \backslash H} z_{F_{1}}-\mathbf{y}^{H \backslash F_{1}} z_{H}\right)+\mathbf{y}^{F_{1} \cap\left(H \backslash F_{2}\right)} \mathbf{y}^{H \backslash F_{1}} z_{H}-\mathbf{y}^{F_{2} \cap\left(H \backslash F_{1}\right)} \mathbf{y}^{F_{2} \backslash H} z_{F_{2}} \\
& =\mathbf{y}^{F_{1} \cap\left(H \backslash F_{2}\right)} b_{F_{1}, H}+\mathbf{y}^{F_{2} \cap\left(H \backslash F_{1}\right)} \mathbf{y}^{H \backslash F_{2}} z_{H}-\mathbf{y}^{F_{2} \cap\left(H \backslash F_{1}\right)} \mathbf{y}^{F_{2} \backslash H} z_{F_{2}} \\
& =\mathbf{y}^{F_{1} \cap\left(H \backslash F_{2}\right)} b_{F_{1}, H}+\mathbf{y}^{F_{2} \cap\left(H \backslash F_{1}\right)} b_{H, F_{2}} .
\end{aligned}
$$

For the rest of the section we let

$$
\mathcal{Q}:=\left\{b_{F_{i}, F_{j}}: \operatorname{dim}\left(F_{i} \cap F_{j}\right)=d-2\right\} .
$$

Observe that the condition $\operatorname{dim}\left(F_{i} \cap F_{j}\right)=d-2$ implies that all binomials in $\mathcal{Q}$ are quadratic. Conversely, for every binomial $b_{F_{i}, F_{j}}$ with $\operatorname{deg}\left(b_{F_{i}, F_{j}}\right)=2$ we have that $\operatorname{dim}\left(F_{i} \cap F_{j}\right)=d-2$. We now establish a technical lemma that will be crucial in the proof of Theorem 6.3.

Lemma 6.7. Fix a term order $<$ on $\mathbb{F}[\mathbf{x}, \mathbf{y}, \mathbf{z}]$ and let $<$ be the associated total order on $\mathcal{F}(\Delta)$. Let $b_{F, F_{1}}, b_{F, F_{2}} \in \mathcal{Q}$ be such that in $\left(b_{F, F_{1}}\right)=y_{F \backslash F_{1}} z_{F}$ and in $\left(b_{F, F_{2}}\right)=y_{F \backslash F_{2}} z_{F}$. Then 


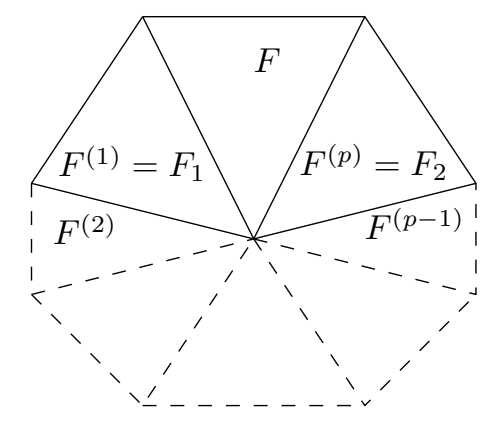

Figure 2. An illustration of Lemma 6.7.

$S\left(b_{F, F_{1}}, b_{F, F_{2}}\right)$ reduces to zero modulo $\mathcal{Q}$ if and only if there exists a sequence of facets $F_{1}=$ $F^{(1)}, \ldots, F^{(p)}=F_{2}$ such that

i. $F_{1} \cap F_{2} \subset F^{(i)}$ for every $1 \leq i \leq p$;

ii. there exists $1 \leq c \leq p$ such that

$$
F^{(1)}>\cdots>F^{(c)}<F^{(c+1)}<\cdots<F^{(p)} .
$$

with $\operatorname{dim}\left(F^{(i)} \cap F^{(i+1)}\right)=d-2$, for every $1 \leq i \leq p-1$.

Proof: $\quad$ The facets $F_{1}$ and $F_{2}$ must meet in codimension one or two, and the proof of Proposition 4.5 yields that $S\left(b_{F, F_{1}}, b_{F, F_{2}}\right)=\mathbf{y}^{\left(F_{1} \cap F_{2}\right) \backslash F} b_{F_{2}, F_{1}}$.

If $F_{1}$ and $F_{2}$ meet in codimension one, then $b_{F_{2}, F_{1}} \in \mathcal{Q}$. In particular, $S\left(b_{F, F_{1}}, b_{F, F_{2}}\right)$ reduces to zero modulo $\mathcal{Q}$, and the sequence of facets consisting of $F_{1}$ and $F_{2}$ always satisfies the hypotheses.

From now on we will hence assume $F_{1}$ and $F_{2}$ meet in codimension two. Then $F_{1} \cap F_{2} \subseteq F$ and thus $S\left(b_{F, F_{1}}, b_{F, F_{2}}\right)=b_{F_{2}, F_{1}}$. Assume without loss of generality that $F_{1}>F_{2}$.

Only if: We assume that $S\left(b_{F, F_{1}}, b_{F, F_{2}}\right)=b_{F_{2}, F_{1}}$ reduces to zero modulo $\mathcal{Q}$ and prove the existence of a sequence of facets satisfying the hypotheses. By hypothesis, $b_{F_{2}, F_{1}} \notin$ $\mathcal{Q}$ becomes zero after applying finitely many reductions via binomials in $\mathcal{Q}$. Let $\left(b_{1}, \ldots, b_{p-1}\right)$ (with $p \geq 3$ ) be the shortest possible sequence of binomials used to reduce $b_{F_{2}, F_{1}}$ to zero. By Lemma $6.6, b_{F_{2}, F_{1}}$ can be reduced via a binomial in $\mathcal{Q}$ if and only if such a binomial is (up to sign) $b_{F_{1}, H_{1}}$, where $H_{1}$ is a facet meeting $F_{1}$ in codimension one and such that $F_{1}>H_{1}$ and $F_{1} \cap F_{2} \subseteq H_{1}$. Moreover, such reduction produces a $y$-multiple of $b_{F_{2}, H_{1}}$; hence, if $p=3$, then $F_{2}$ and $H_{1}$ must meet in codimension one and $\left(F_{1}, H_{1}, F_{2}\right)$ is the desired chain. If instead $p>3$, then $F_{2}$ and $H_{1}$ must meet in codimension two (since $F_{1} \cap F_{2} \subseteq H_{1} \cap F_{2}$ ). Applying Lemma 6.6 to (the given $y$-multiple of) $b_{F_{2}, H_{1}}$, we find another facet $H_{2}$ meeting $\max \left\{H_{1}, F_{2}\right\}$ in codimension one and such that $\max \left\{H_{1}, F_{2}\right\}>H_{2}$ and $H_{1} \cap F_{2} \subseteq H_{2}$. Moreover, one also has that $F_{1} \cap F_{2} \subseteq H_{1} \cap F_{2} \subseteq H_{2}$, as requested. The reduction step produces a $y$-multiple of $b_{\min \left\{H_{1}, F_{2}\right\}, H_{2}}$; reasoning as before, if $p=4$ then $\min \left\{H_{1}, F_{2}\right\}$ and $H_{2}$ must meet in codimension one and we are done, otherwise we continue the process until we reach the end of the reducing sequence. 
If: For the reverse implication, assume that we are given a sequence of facets $F^{(1)}>$ $\cdots>F^{(c)}<F^{(c+1)}<\cdots<F^{(p)}$ satisfying the hypotheses. Then one gets the desired sequence of binomials by the following algorithm:

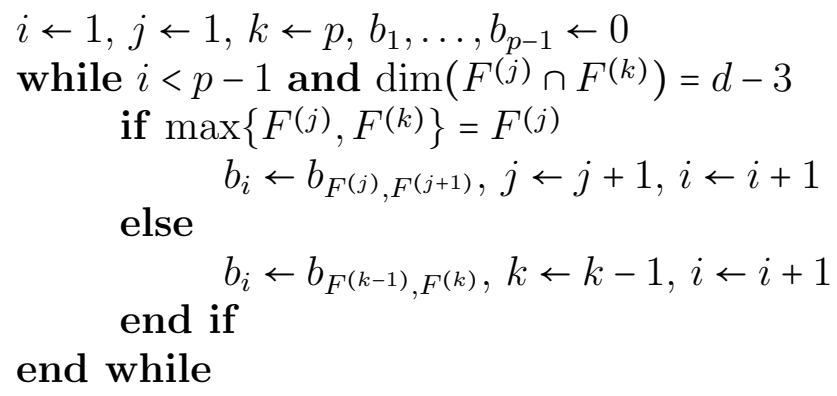

Note that we are invoking Lemma 6.6 at every iteration inside the while cycle. This can be done because, whenever $F^{(j)}$ and $F^{(k)}$ meet in codimension two, then $F^{(j)} \cap$ $F^{(k)}=F_{1} \cap F_{2}$, and $F_{1} \cap F_{2}$ is contained both in $F^{(j+1)}$ and $F^{(k-1)}$ by hypothesis. Moreover, when the condition $\operatorname{dim}\left(F^{(j)} \cap F^{(k)}\right)=d-3$ is not met, then it must be that $F^{(j)}$ and $F^{(k)}$ meet in codimension one, and thus we can stop the process after a final reduction via $b_{F^{(j)}, F^{(k)}}$.

We are now ready to prove the main result in this section.

Proof of Theorem 6.3 We prove the two implications separately.

Only if: Let $F_{1}<\cdots<F_{|\mathcal{F}(\Delta)|}$ be a shelling order for $\Delta$. Then the collection

$$
\begin{aligned}
C_{\Delta}= & \mathcal{Q} \cup\left\{x_{i} y_{i}: i=1, \ldots, n\right\} \cup\left\{\mathbf{x}^{N_{i}}: N_{i} \text { minimal nonface of } \Delta\right\} \cup \\
& \left\{z_{F_{i}} z_{F_{j}}: F_{i}, F_{j} \text { facets of } \Delta\right\} \cup\left\{x_{i} z_{F_{j}}: F_{j} \text { facet of } \Delta, i \notin F_{j}\right\}
\end{aligned}
$$

is a Gröbner basis for the ideal defining $R_{\Delta}$ with respect to any term order compatible with $\prec$.

Since $\Delta$ is flag, all monomials in the collection above have degree 2. Moreover, shellable complexes are $\left(S_{2}\right)$, and hence the algebra $R_{\Delta}$ is quadratic by Proposition 4.4. In particular, the defining ideal of $R_{\Delta}$ is generated by $C_{\Delta}$, as this is the set of degree 2 elements of the generating set described in Proposition 4.3. It is left to the reader to check that all the $S$ polynomials obtained by comparing a monomial and a binomial in $C_{\Delta}$ reduce to zero. Hence, to prove the claim we only need to show that the $S$-polynomials of the form $S\left(b_{1}, b_{2}\right)$, with $b_{1}, b_{2} \in \mathcal{Q}$, reduce to zero. Observe that with the term order we fixed, the leading term of each binomial is determined by the $z$-variables alone. We begin by noting that if two binomials $b_{1}$ and $b_{2}$ are such that $\operatorname{in}\left(b_{1}\right)=y_{i} z_{F}$ and $\operatorname{in}\left(b_{2}\right)=y_{j} z_{G}$, with $F \neq G$, then $S\left(b_{1}, b_{2}\right)$ reduces to zero, even if $i=j$. This follows from the fact that both monomials of $S\left(b_{1}, b_{2}\right)$ contain a product of two $z$-variables. Since the collection $C_{\Delta}$ contains all monomials of degree 2 in the $z$-variables, these $S$-polynomials reduce to zero. 
Consider now $S\left(b_{F_{k}, F_{\ell}}, b_{F_{k}, F_{m}}\right)$, with $k>\ell$ and $k>m$. By Lemma 6.7, the polynomial $S\left(b_{F_{k}, F_{\ell}}, b_{F_{k}, F_{m}}\right)=\mathbf{y}^{\left(F_{\ell} \cap F_{m}\right) \backslash F_{k}} b_{F_{m}, F_{\ell}}$ reduces to zero modulo the collection $C_{\Delta}$ if and only if it reduces to zero modulo the collection $C_{\Delta_{k}}$, with $\Delta_{k}=\left\langle F_{1}, \ldots, F_{k}\right\rangle$.

Let $H=F_{\ell} \cap F_{m} \in \Delta_{k-1}$ and note that $|H| \geq d-2$.

If $|H|=d-1$, then $b_{F_{\ell}, F_{m}} \in \mathcal{Q}$, which implies that $S\left(b_{F_{k}, F_{\ell}}, b_{F_{k}, F_{m}}\right)$ is a multiple of a binomial in $\mathcal{Q}$. In particular, $S\left(b_{F_{k}, F_{\ell}}, b_{F_{k}, F_{m}}\right)$ reduces to zero.

Assume then that $|H|=d-2$. Since $\Delta_{k-1}$ is shellable, the 1-dimensional simplicial complex $\mathrm{lk}_{\Delta_{k-1}}(H)$ is shellable, with a shelling order induced by the one of $\Delta_{k-1}$. Since shellable simplicial complexes of dimension greater than zero are connected, there exists a path in $\mathrm{lk}_{\Delta_{k-1}}(H)$ connecting the two edges $F_{\ell} \backslash H$ and $F_{m} \backslash H$. The edges of this path correspond to facets of $\Delta_{k-1}$ via taking their union with $H$ : therefore, we can identify the path with a sequence of facets of $\Delta_{k-1}$ which all contain $H$. We can describe the support of such a path via a binary string of length $\left|\mathcal{F}\left(\Delta_{k-1}\right)\right|$ whose $i$-th character is 1 if $F_{i} \in P$ and 0 if $F_{i} \notin P$. We consider the colexicographic order on these strings, i.e.,

$$
\left(s_{1}, \ldots, s_{\left|\mathcal{F}\left(\Delta_{k-1}\right)\right|}\right)<_{\text {colex }}\left(t_{1}, \ldots, t_{\left|\mathcal{F}\left(\Delta_{k-1}\right)\right|}\right) \Longleftrightarrow s_{i}<t_{i} \text { with } i=\max \left\{j: s_{j} \neq t_{j}\right\} .
$$

It is important to observe that the path between $F_{\ell}$ and $F_{m}$ might not be unique. We choose the one $P: F_{\ell}=F_{i_{1}} \rightarrow F_{i_{2}} \cdots \rightarrow F_{i_{p}}=F_{m}$ whose support is minimal with respect to the colexicographic order on $2^{\mathcal{F}\left(\Delta_{k-1}\right)}$. We claim there exists $1 \leq c \leq p$ such that $z_{F_{i_{1}}}>z_{F_{i_{2}}}>\cdots>$ $z_{F_{i_{c}}}<z_{F_{i_{c+1}}}<\cdots<z_{F_{i_{p}}}$.

Assume by contradiction there exist $z_{F_{i_{r}}}<z_{F_{i_{s}}}>z_{F_{i_{t}}}$, with $r<s<t$. The minimality of $P$ implies that one step before the facet $F_{i_{s}} \backslash H$ of $\operatorname{lk}_{\Delta_{k-1}}(H)$ is added via a shelling, there is no path joining the edges corresponding to $F_{i_{r}}$ and $F_{i_{t}}$, which contradicts the connectedness of shellable complexes. Indeed, if such a path existed, we would be able to replace $\left\{F_{i_{r+1}}, \ldots, F_{i_{s}}, \ldots, F_{i_{t-1}}\right\}$ in the support of $P$ with a subset containing only facets which are older than $F_{i_{s}}$ : this would lead to a set colexicographically smaller than the support of $P$, which is impossible. By Lemma $6.7, S\left(b_{F_{k}, F_{\ell}}, b_{F_{k}, F_{m}}\right)$ reduces to zero modulo $C_{\Delta}$.

If: If $R_{\Delta}$ has a quadratic Gröbner basis with respect to $<$, then the total order $<$ on $\mathcal{F}(\Delta)$ induced by $<$ is a shelling order for $\Delta$.

Assume by contradiction that the total order induced by $<$ is not a shelling order, and let $F_{1}<\cdots<F_{|\mathcal{F}(\Delta)|}$ be the facets in this order. This implies that there exists $2 \leq i \leq|\mathcal{F}(\Delta)|$ such that $\Delta_{i-1} \cap\left\langle F_{i}\right\rangle$ is not pure and $(d-2)$-dimensional. In particular, there is a facet $G$ of $\Delta_{i-1} \cap\left\langle F_{i}\right\rangle$ with $\operatorname{dim}(G) \leq d-3$. We observe that $\mathrm{lk}_{\Delta_{i}}(G)$ is not connected in codimension 1. Indeed, $G$ is not properly contained in any face of $\Delta_{i-1} \cap\left\langle F_{i}\right\rangle$, so no face in $\mathrm{lk}_{\Delta_{i}}(G)$ contains vertices of $\Delta_{i-1} \cap\left\langle F_{i}\right\rangle$. In particular, the facet $F_{i} \backslash G$ does not intersect any other facet of $\mathrm{lk}_{\Delta_{i}}(G)$. However, since $R_{\Delta}$ has a quadratic Gröbner basis then it is Koszul, and hence by Corollary $5.3 \Delta$ is Cohen-Macaulay. In particular, $\operatorname{lk}_{\Delta}(G)$ is Cohen-Macaulay, and hence it is connected in codimension 1. This implies that $i<|\mathcal{F}(\Delta)|$. Let $k$ be the smallest integer in $\{i+1, \ldots,|\mathcal{F}(\Delta)|\}$ such that $\mathrm{lk}_{\Delta_{k}}(G)$ is connected in codimension 1 . Then there exist $1 \leq r, s \leq k-1$ such that

- $G \subset F_{r}, G \subset F_{s}$;

- $\left(F_{k} \backslash G\right) \cap\left(F_{r} \backslash G\right)$ and $\left(F_{k} \backslash G\right) \cap\left(F_{s} \backslash G\right)$ are faces of codimension 1 of $\mathrm{lk}_{\Delta_{k}}(G)$;

- $\left(F_{r} \backslash G\right)$ and $\left(F_{s} \backslash G\right)$ are not connected in codimension 1 in $\operatorname{lk}_{\Delta_{k-1}}(G)$.

Hence $\operatorname{dim}\left(F_{k} \cap F_{r}\right)=\operatorname{dim}\left(F_{k} \cap F_{s}\right)=d-2$, which implies that $b_{F_{k}, F_{r}}, b_{F_{k}, F_{s}} \in \mathcal{Q}$. 
We claim that $S\left(b_{F_{k}, F_{r}}, b_{F_{k}, F_{s}}\right)$ does not reduce to zero modulo the set $\mathcal{Q}$. By Lemma 6.7, the reduction to zero of this polynomial implies the existence of a sequence of facets $F_{r}=$ $F^{(1)} \ldots, F^{(p)}=F_{s}$ in $\Delta_{k-1}$ such that

- $F^{(t)} \leq \max \left\{F_{r}, F_{s}\right\}$ for every $1 \leq t \leq p$;

- $G \subset F_{r} \cap F_{s} \subset F^{(t)}$ for every $1 \leq t \leq p$;

- $\operatorname{dim}\left(F^{(t)} \cap F^{(t+1)}\right)=d-2$ for every $1 \leq t \leq p-1$.

The first condition implies that $F^{(t)} \in \Delta_{k-1}$, the second yields that $F^{(t)} \backslash G$ is a facet of $\mathrm{lk}_{\Delta_{k-1}}(G)$, while the third implies the existence of a path of facets of $\operatorname{lk}_{\Delta_{k-1}}(G)$ connecting $F_{r} \backslash G$ and $F_{s} \backslash G$. However, such a sequence does not exist, as $F_{r} \backslash G$ and $F_{s} \backslash G$ are not connected in codimension 1 in $\operatorname{lk}_{\Delta_{k-1}}(G)$. This proves that if $\Delta$ is not shellable, then the set $C_{\Delta}$ is not a Gröbner basis. To conclude that $R_{\Delta}$ has no quadratic Gröbner basis we use the fact that set $\mathcal{U}$ given in Proposition 4.3 is a universal Gröbner basis for $R_{\Delta}$ by Proposition 4.5, and hence every quadratic reduced Gröbner basis must be a subset of the set of quadratic polynomials in $\mathcal{U}$, which is precisely $C_{\Delta}$.

Example 6.8. Let $\Delta=\langle 123,234,345\rangle$ as in Example 4.6. Up to sign, the only two binomials in $\mathcal{Q}$ are

$$
f=y_{4} z_{234}-y_{1} z_{123} \text { and } g=y_{5} z_{345}-y_{2} z_{234} .
$$

If we consider a term order compatible with the shelling order $123<234<345$, then we have $\operatorname{in}(f)=y_{4} z_{234}$ and $\operatorname{in}(g)=y_{5} z_{345}$. Since the two leading terms are coprime in the $z$-variables, $S(f, g)$ reduces to zero modulo the minimal generating set of $\left(z_{123}, z_{234}, z_{345}\right)^{2}$. On the other hand, if we consider a term order compatible with $123<345<234$, which is not a shelling order, we obtain $\operatorname{in}(f)=y_{4} z_{234}$ and $\operatorname{in}(g)=y_{2} z_{234}$. In this case we see that none of the monomials in $S(f, g)=y_{4} y_{5} z_{345}-y_{1} y_{2} z_{123}$ is divisible by any of the leading terms of the elements of $\mathcal{Q}$. Hence, $S(f, g)$ does not reduce to zero.

\section{THE $\gamma$-VECTOR OF $R_{\Delta}$}

The aim of this section is to study the $\gamma$-vector of the algebra $R_{\Delta}$ in terms of combinatorial properties of the simplicial complex $\Delta$. In general, the $\gamma$-vector is often the "right" invariant to consider when we want to highlight the information encoded in a palindromic vector, like for instance the $h$-vector of a simplicial sphere. See [Brä06], [Gal05], and [Ath18] for more information on this topic.

In particular, recall that the coefficients of the $h$-polynomial of a standard graded Gorenstein algebra form a palindromic sequence. More precisely, if the degree of the $h$-polynomial (which coincides with the Castelnuovo-Mumford regularity since the algebra is Cohen-Macaulay) equals $s$, we have that $h_{i}=h_{s-i}$ for every $i$. The vector space of univariate palindromic polynomials of degree $s$ has dimension $\left\lfloor\frac{s}{2}\right\rfloor+1$, and Gal proposed the use of the basis $\left\{t^{i}(t+\right.$ $\left.1)^{s-2 i}\right\}_{i=0}^{\left\lfloor\frac{s}{2}\right\rfloor}[$ Gal05, Section 2].

Definition 7.1. The $\gamma$-vector associated with the integer vector $h=\left(h_{0}, \ldots, h_{s}\right)$ with $h_{i}=$ $h_{s-i}$ is the integer vector $\gamma=\left(\gamma_{0}, \ldots, \gamma_{\left\lfloor\frac{s}{2}\right\rfloor}\right)$ defined by the identity

$$
\sum_{i=0}^{\left\lfloor\frac{s}{2}\right\rfloor} \gamma_{i} t^{i}(t+1)^{s-2 i}=\sum_{i=0}^{s} h_{i} t^{i} .
$$


We denote by $\gamma(A)$ the $\gamma$-vector associated with the $h$-vector of a standard graded Gorenstein algebra $A$.

Each $\gamma_{i}$ can be expressed as an integer linear combination of the $h_{i}$ : for instance, one has that $\gamma_{0}=h_{0}, \gamma_{1}=h_{1}-s h_{0}$ and $\gamma_{2}=h_{2}-(s-2) h_{1}+\frac{s(s-3)}{2} h_{0}$. In general, the following recursive formula holds:

$$
\gamma_{i}=h_{i}-\sum_{j=0}^{i-1}\left(\begin{array}{c}
s-2 j \\
i-j
\end{array}\right) \gamma_{j}
$$

For the rest of the section we will focus on the $\gamma$-vector of the standard graded Gorenstein algebra $R_{\Delta}$, with $\Delta$ a pure simplicial complex. If $\Delta$ is $(d-1)$-dimensional, then the $h$ polynomial of $R_{\Delta}$ has degree $d+1$, and we will then replace $s$ with $d+1$ in Definition 7.1. Perhaps surprisingly, we will show that when $R_{\Delta}$ is Koszul over $\mathbb{F}$ - i.e., by Corollary 5.3, when $\Delta$ is Cohen-Macaulay over $\mathbb{F}$ - many of the $\gamma_{i}$ are nonpositive.

We begin with a result on the last entry of the $\gamma$-vector. Even though we will prove a more general result in Corollary 7.10, it is instructive to consider this special case separately, as its proof is rather elementary.

Lemma 7.2. For any $(d-1)$-dimensional Cohen-Macaulay simplicial complex $\Delta$, with $d \geq 3$ odd, we have that

$$
\gamma_{\frac{d+1}{2}}\left(R_{\Delta}\right)=(-1)^{\frac{d-1}{2}} 2 \widetilde{\chi}(\Delta)=(-1)^{\frac{d-1}{2}} 2 \operatorname{dim}_{\mathbb{F}} \widetilde{H}_{d-1}(\Delta ; \mathbb{F}) .
$$

In particular, if $d \equiv 3 \bmod 4$, then $\gamma_{\frac{d+1}{2}}\left(R_{\Delta}\right)$ is nonpositive; moreover, for any integer $c \geq 0$ and every $d \equiv 3 \bmod 4$, there exists a Cohen-Macaulay (even shellable) $(d-1)$-complex $\Delta$ such that $\gamma_{\frac{d+1}{2}}\left(R_{\Delta}\right)=-2$ c. Such a complex can be chosen to be flag.

Proof: $\quad$ Let $f(\Delta)=\left(1, f_{0}, \ldots, f_{d-1}\right)$ be the $f$-vector of $\Delta$ and recall that, as noted in Remark 4.2, $h\left(R_{\Delta}\right)=\left(1, f_{0}+f_{d-1}, f_{1}+f_{d-2}, \ldots, f_{0}+f_{d-1}, 1\right)$. Evaluating $(7.1)$ at $t=-1$ yields

$$
(-1)^{\frac{d+1}{2}} \gamma_{\frac{d+1}{2}}\left(R_{\Delta}\right)=\sum_{i=0}^{d+1}(-1)^{i} h_{i}\left(R_{\Delta}\right)=2+\sum_{i=1}^{d}(-1)^{i}\left(f_{i-1}+f_{d-i}\right)=-2 \widetilde{\chi}(\Delta) .
$$

It is a standard fact from algebraic topology that the reduced Euler characteristic can be written as the alternating sum of the dimensions of the reduced homology groups of $\Delta$. Since $\Delta$ is Cohen-Macaulay, one has that $\widetilde{H}_{j}(\Delta ; \mathbb{F})=0$ for every $0 \leq j<d-1$, and thus $\gamma_{\frac{d+1}{2}}\left(R_{\Delta}\right)=(-1)^{\frac{d-1}{2}} 2 \operatorname{dim}_{\mathbb{F}} \widetilde{H}_{d-1}(\Delta ; \mathbb{F})$.

To prove the last claim it is enough to exhibit a $(d-1)$-dimensional Cohen-Macaulay flag complex with $\tilde{\chi}(\Delta)=c$. For instance, we can glue together $c$ copies of the boundary complex of the $d$-dimensional cross-polytope so that they all share the same single facet. It is elementary to check that this simplicial complex has the desired properties, and that it is indeed even shellable.

In their collection [PS09] of problems on syzygies and Hilbert functions, Peeva and Stillman suggest that it might make sense to consider a Charney-Davis-like conjecture for (not necessarily monomial) Koszul Gorenstein algebras:

Question 7.3 ([PS09, Problem 10.3]). Let S/I be a Koszul Gorenstein algebra with h-vector $\left(h_{0}, h_{1}, \ldots, h_{2 e}\right)$. Is it true that $(-1)^{e}\left(h_{0}-h_{1}+h_{2}-\ldots+h_{2 e}\right) \geq 0$ ? 
Notice that $(-1)^{e}\left(h_{0}-h_{1}+h_{2}-\ldots+h_{2 e}\right)=\gamma_{e}(S / I)$. Lemma 7.2 and Corollary 5.3 immediately yield a negative answer to Question 7.3:

Corollary 7.4. Let $d \equiv 3 \bmod 4, c \in \mathbb{Z}_{+}$. Then there exists a Koszul Gorenstein algebra with $h$-vector $\left(h_{0}, h_{1}, \ldots, h_{d+1}\right)$ and such that $(-1)^{\frac{d+1}{2}}\left(h_{0}-h_{1}+h_{2}-\ldots+h_{2 e}\right)=-2 c<0$. Such an algebra is of the form $R_{\Delta}$, where $\Delta$ is a flag Cohen-Macaulay $(d-1)$-dimensional complex with $\operatorname{dim}_{\mathbb{F}} \widetilde{H}_{d-1}(\Delta ; \mathbb{F})=c$.

Example 7.5. Let $\Delta$ be the boundary of the 3-dimensional cross-polytope (or octahedron). Since $f(\Delta)=(1,6,12,8)$, one has that $h\left(R_{\Delta}\right)=(1,14,24,14,1)$ and hence $\gamma_{2}\left(R_{\Delta}\right)=1-14+$ $24-14+1=-2<0$. Labeling the antipodal pairs of vertices of $\Delta$ by $\{1,2\},\{3,4\}$ and $\{5,6\}$ yields that $R_{\Delta}$ is presented as the quotient of the polynomial ring in $2 f_{0}(\Delta)+f_{2}(\Delta)=20$ variables

$$
\mathbb{F}\left[x_{1}, x_{2}, x_{3}, x_{4}, x_{5}, x_{6}, y_{1}, y_{2}, y_{3}, y_{4}, y_{5}, y_{6}, z_{135}, z_{136}, z_{145}, z_{146}, z_{235}, z_{236}, z_{245}, z_{246}\right]
$$

by the ideal with 81 quadratic generators

$$
\begin{aligned}
& \left(x_{1} x_{2}, x_{3} x_{4}, x_{5} x_{6}, x_{1} y_{1}, x_{2} y_{2}, x_{3} y_{3}, x_{4} y_{4}, x_{5} y_{5}, x_{6} y_{6}\right) \\
+ & \left(z_{135}, z_{136}, z_{145}, z_{146}, z_{235}, z_{236}, z_{245}, z_{246}\right)^{2} \\
+ & \left(x_{2} z_{135}, x_{4} z_{135}, x_{6} z_{135}, x_{2} z_{136}, x_{4} z_{136}, x_{5} z_{136}, x_{2} z_{145}, x_{3} z_{145}, x_{6} z_{145}, x_{2} z_{146}, x_{3} z_{146}, x_{5} z_{146},\right. \\
& \left.\quad x_{1} z_{235}, x_{4} z_{235}, x_{6} z_{235}, x_{1} z_{236}, x_{4} z_{236}, x_{5} z_{236}, x_{1} z_{245}, x_{3} z_{245}, x_{6} z_{245}, x_{1} z_{246}, x_{3} z_{246}, x_{5} z_{246}\right) \\
+ & \left(y_{5} z_{135}-y_{6} z_{136}, y_{3} z_{135}-y_{4} z_{145}, y_{1} z_{135}-y_{2} z_{235}, y_{3} z_{136}-y_{4} z_{146}, y_{1} z_{136}-y_{2} z_{236}, y_{5} z_{145}-y_{6} z_{146},\right. \\
& \left.y_{1} z_{145}-y_{2} z_{245}, y_{1} z_{146}-y_{2} z_{246}, y_{5} z_{235}-y_{6} z_{236}, y_{3} z_{235}-y_{4} z_{245}, y_{3} z_{236}-y_{4} z_{246}, y_{5} z_{245}-y_{6} z_{246}\right) .
\end{aligned}
$$

Being the boundary complex of a simplicial polytope, the simplicial complex $\Delta$ is shellable. Therefore, by Theorem 6.3 the 81 generators listed above are a Gröbner basis with respect to any term order compatible with any shelling order. We remark that, by Propositions 8.1 and 8.3, one can get an analogous Artinian example with 14 variables by going modulo the regular sequence of linear forms $y_{1}-x_{1}, \ldots, y_{6}-x_{6}$.

Remark 7.6. Example 7.5 also provides a Koszul Gorenstein algebra which is not PF (in the sense of [RW05]) and has Castelnuovo-Mumford regularity 4. By [RW05, Corollary 4.14], all Koszul Gorenstein algebras of regularity at most 3 are PF and hence have the Charney-Davis (CD) property.

Example 7.7. Let $\Delta$ be the flag triangulation of $\mathbb{R P}^{2}$ described in Example 5.16. We have observed that $h\left(R_{\Delta}\right)=(1,31,60,31,1)$, and hence $\gamma_{2}\left(R_{\Delta}\right)=0$. This is in line with Lemma 7.2 , as $\operatorname{dim}_{\mathbb{F}} \widetilde{H}_{2}\left(\mathbb{R P}^{2} ; \mathbb{F}\right)=0$ for every field $\mathbb{F}$ over which $\Delta$ is Cohen-Macaulay, i.e., every field of characteristic different than 2.

For the rest of the section we stipulate that a binomial coefficient $\left(\begin{array}{l}n \\ k\end{array}\right)$ is equal to zero whenever $k<0$ or $n<k$. Moreover, whenever $r \geq 2 i \geq 0$, we set

$$
\ell_{r, i}:= \begin{cases}2 & \text { if }(r, i)=(0,0) \\
\left(\begin{array}{c}
r-i \\
i
\end{array}\right)+\left(\begin{array}{c}
r-i-1 \\
i-1
\end{array}\right)=\frac{r}{r-i}\left(\begin{array}{c}
r-i \\
i
\end{array}\right) & \text { otherwise. }\end{cases}
$$


The positive integers $\ell_{r, i}$ appear as coefficients of Lucas polynomials $^{1}$ in sequence A034807 of the On-Line Encyclopedia of Integer Sequences [Inc21].

We begin with a technical lemma.

Lemma 7.8. The following identities hold for any $n, m, r \geq 0$ :

$i$.

$$
1+t^{r}=\sum_{i=0}^{\left\lfloor\frac{r}{2}\right\rfloor}(-1)^{i} \ell_{r, i} t^{i}(1+t)^{r-2 i}
$$

ii.

$$
\left(\begin{array}{l}
n \\
m
\end{array}\right)+\left(\begin{array}{c}
n \\
m-r
\end{array}\right)=\sum_{i=0}^{\left\lfloor\frac{r}{2}\right\rfloor}(-1)^{i} \ell_{r, i}\left(\begin{array}{c}
n+r-2 i \\
m-i
\end{array}\right) .
$$

Proof: $\quad$ Statement $i$ is a specialization of a classical identity due to Girard and Waring [Gou99, Identity 1] which can be derived from the Newton formulas relating power sums and elementary symmetric polynomials.

Expanding $(1+t)^{r-2 i}$ inside identity $i$ yields that

$$
1+t^{r}=\sum_{i=0}^{\left\lfloor\frac{r}{2}\right\rfloor} \sum_{j=0}^{r-2 i}(-1)^{i} \ell_{r, i}\left(\begin{array}{c}
r-2 i \\
j
\end{array}\right) t^{i+j}
$$

This is now an identity inside the free $\mathbb{Z}$-module with basis $\left\{1, t, t^{2}, \ldots, t^{r}\right\}$. We can now specialize this by substituting each $t^{i}$ with the binomial coefficient $\left(\begin{array}{c}n \\ m-i\end{array}\right)$, obtaining

$$
\left(\begin{array}{c}
n \\
m
\end{array}\right)+\left(\begin{array}{c}
n \\
m-r
\end{array}\right)=\sum_{i=0}^{\left\lfloor\frac{r}{2}\right\rfloor}(-1)^{i} \ell_{r, i}\left(\sum_{j=0}^{r-2 i}\left(\begin{array}{c}
r-2 i \\
j
\end{array}\right)\left(\begin{array}{c}
n \\
m-i-j
\end{array}\right)\right) .
$$

Applying Vandermonde's identity for binomial coefficients yields identity $i i$.

Proposition 7.9. Let $\Delta$ be $a(d-1)$-dimensional pure simplicial complex. Then

$$
\gamma_{i}\left(R_{\Delta}\right)=(-1)^{i-1} \sum_{k=2 i-1}^{d} \ell_{k-1, i-1} h_{k}(\Delta)
$$

for every $i \geq 1$. In particular, each $\gamma_{i}\left(R_{\Delta}\right)$ is $(-1)^{i-1}$ times a linear combination of the $h_{k}(\Delta)$ with positive integer coefficients.

Proof: Throughout this proof we will set $\gamma_{j}:=\gamma_{j}\left(R_{\Delta}\right), h_{j}:=h_{j}(\Delta)$ and $f_{j}:=f_{j}(\Delta)$. We prove the desired formula for $\gamma_{i}$ by induction on $i \geq 1$.

For the base case $i=1$ it suffices to observe that $\gamma_{1}=h_{1}\left(R_{\Delta}\right)-d-1=f_{0}+f_{d-1}-d-1=$ $2 h_{1}+\sum_{k=2}^{d} h_{k}$, where we used (2.4) to express $f_{0}$ and $f_{d-1}$ as functions of the $h_{j}$.

Let us now fix $i>1$ and assume that the claim holds for every $1 \leq j<i$. (7.2) gives us a way to express $\gamma_{i}$ as a function of the $\gamma_{j}$ with $j<i$; moreover, using again (2.4), we can explicitly write each $h_{i}\left(R_{\Delta}\right)=f_{i-1}+f_{d-i}$ as a combination of the $h_{i}=h_{i}(\Delta)$. Putting together these two

\footnotetext{
${ }^{1}$ There appear to be at least two different families of polynomials known as Lucas polynomials, but in both cases the nonzero coefficients are the integers $\ell_{r, i}$.
} 
facts with the inductive hypothesis on the $\gamma_{j}$ with $j<i$ and the observation that $\gamma_{0}=h_{0}=1$, we can write

$$
\begin{aligned}
\gamma_{i} & =h_{i}\left(R_{\Delta}\right)-\sum_{j=0}^{i-1}\left(\begin{array}{c}
d+1-2 j \\
i-j
\end{array}\right) \gamma_{j} \\
& =f_{i-1}+f_{d-i}-\left(\begin{array}{c}
d+1 \\
i
\end{array}\right) \gamma_{0}-\sum_{j=1}^{i-1}\left(\begin{array}{c}
d+1-2 j \\
i-j
\end{array}\right) \gamma_{j} \\
& =\sum_{k=0}^{i}\left(\begin{array}{c}
d-k \\
d-i
\end{array}\right) h_{k}+\sum_{k=0}^{d-i+1}\left(\begin{array}{c}
d-k \\
i-1
\end{array}\right) h_{k}-\left(\begin{array}{c}
d+1 \\
i
\end{array}\right) h_{0}-\sum_{j=0}^{i-2}\left(\begin{array}{c}
d-1-2 j \\
i-j-1
\end{array}\right) \gamma_{j+1} \\
& =\sum_{k=0}^{i}\left(\begin{array}{c}
d-k \\
d-i
\end{array}\right) h_{k}+\sum_{k=0}^{d-i+1}\left(\begin{array}{c}
d-k \\
i-1
\end{array}\right) h_{k}-\left(\begin{array}{c}
d+1 \\
i
\end{array}\right) h_{0}-\sum_{j=0}^{i-2}\left(\begin{array}{c}
d-1-2 j \\
i-j-1
\end{array}\right)(-1)^{j} \sum_{s=2 j+1}^{d} \ell_{s-1, j} h_{s} .
\end{aligned}
$$

We have thus obtained an expression for $\gamma_{i}$ as a linear combination of the $h_{j}$. We will denote by $\left[\gamma_{i}\right]_{k}$ the coefficient of $h_{k}$ in this expression and will analyze such coefficients separately. One has immediately that $\left[\gamma_{i}\right]_{0}=\left(\begin{array}{c}d \\ i\end{array}\right)+\left(\begin{array}{c}d \\ i-1\end{array}\right)-\left(\begin{array}{c}d+1 \\ i\end{array}\right)=0$. When $0<k \leq d$, we obtain

$$
\left[\gamma_{i}\right]_{k}=\left(\begin{array}{c}
d-k \\
d-i
\end{array}\right)+\left(\begin{array}{c}
d-k \\
i-1
\end{array}\right)-\sum_{j=0}^{\min \left\{i-2,\left\lfloor\frac{k-1}{2}\right\rfloor\right\}}\left(\begin{array}{c}
d-1-2 j \\
i-j-1
\end{array}\right)(-1)^{j} \ell_{k-1, j}
$$

We wish to rewrite $\left(\begin{array}{c}d-k \\ d-i\end{array}\right)+\left(\begin{array}{c}d-k \\ i-1\end{array}\right)$ in the above expression. Noting that $\left(\begin{array}{c}d-k \\ d-i\end{array}\right)=\left(\begin{array}{c}d-k \\ i-1-(k-1)\end{array}\right)$, we are in the position to apply Lemma 7.8.ii with $n=d-k, m=i-1$ and $r=k-1$, obtaining that

$$
\left(\begin{array}{c}
d-k \\
d-i
\end{array}\right)+\left(\begin{array}{c}
d-k \\
i-1
\end{array}\right)=\sum_{j=0}^{\left\lfloor\frac{k-1}{2}\right\rfloor}\left(\begin{array}{c}
d-1-2 j \\
i-j-1
\end{array}\right)(-1)^{j} \ell_{k-1, j} .
$$

Hence, (7.3) immediately yields that $\left[\gamma_{i}\right]_{k}=0$ whenever $\min \left\{i-2,\left\lfloor\frac{k-1}{2}\right\rfloor\right\}=\left\lfloor\frac{k-1}{2}\right\rfloor$, which happens when $k \leq 2 i-2$.

Now assume that $2 i-1 \leq k \leq d$. Then $\min \left\{i-2,\left\lfloor\frac{k-1}{2}\right\rfloor\right\}=i-2$, and so (7.3) becomes

$$
\left[\gamma_{i}\right]_{k}=\sum_{j=i-1}^{\left\lfloor\frac{k-1}{2}\right\rfloor}\left(\begin{array}{c}
d-1-2 j \\
i-j-1
\end{array}\right)(-1)^{j} \ell_{k-1, j}=(-1)^{i-1} \ell_{k-1, i-1}
$$

with the last equality holding since the binomial coefficient $\left(\begin{array}{c}d-1-2 j \\ i-j-1\end{array}\right)$ equals 1 when $j=i-1$ and vanishes otherwise.

Corollary 7.10. Let $\Delta$ be a $(d-1)$-dimensional simplicial complex for which $h_{i}(\Delta) \geq 0$ holds for every $0 \leq i \leq d$. Then

$$
(-1)^{i-1} \gamma_{i}\left(R_{\Delta}\right) \geq 0
$$

for every $1 \leq i \leq\left\lfloor\frac{d+1}{2}\right\rfloor$.

In particular, the algebra $R_{\Delta}$ has $\gamma$-numbers which alternate in sign whenever $\Delta$ is CohenMacaulay. However, the hypothesis in Corollary 7.10 is satisfied by larger families of simplicial complexes, such as partitionable complexes. 
Remark 7.11. We would like to stress that the formula in Proposition 7.9 is of purely combinatorial nature, and it holds for more general integer vectors than $f$-and $h$-vectors of simplicial complexes. Let $\mathfrak{a}=\left(a_{0}, a_{1}, \ldots, a_{d+1}\right)$ be any sequence of integers with $a_{i}=a_{d+1-i}$, and let $\mathfrak{b}=\left(b_{0}, b_{1}, \ldots, b_{d}\right)$ be a sequence which satisfies $b_{0}=a_{0}$ and $b_{i}+b_{d+1-i}=a_{i}$, for every $0<i \leq d$. Proposition 7.9 allows to express the $\gamma$-vector of $\mathfrak{a}$ as a linear combination of the entries of a vector $\mathfrak{c}$, obtained from $\mathfrak{b}$ via the linear transformation (2.3).

\section{An Artinian Reduction of $R_{\Delta}$ And a CONnection to Work of Gondim And ZAPPALÀ}

After the first version of this paper was completed, we noticed a connection between the algebras $R_{\Delta}$ defined here and the Artinian Gorenstein algebras $A_{\Delta}$ introduced by Gondim and Zappalà in their paper [GZ18]: more specifically, $A_{\Delta}$ is an Artinian reduction of $R_{\Delta}$ in characteristic zero. The aim of this final section is to clarify this connection.

For this section, let the characteristic of the field $\mathbb{F}$ be zero ${ }^{2}$. Under this assumption, it is known that every graded Artinian Gorenstein algebra corresponds to a single homogeneous polynomial $f$ via Macaulay's inverse system (see e.g. [IK99, Section 0.2]). The key idea of the paper by Gondim and Zappalà is the following: given a pure simplicial complex $\Delta$ with $n$ vertices, consider the polynomial ring $\mathbb{F}\left[x_{i}, z_{F} \mid i \in\{1, \ldots, n\}, F\right.$ facet of $\left.\Delta\right]$ and let

$$
f_{\Delta}:=\sum_{F \text { facet of } \Delta} z_{F} \cdot \prod_{i \in F} x_{i} .
$$

Call $A_{\Delta}$ the Artinian Gorenstein algebra corresponding to $f_{\Delta}$ via Macaulay's inverse system. After unifying the notation (as the $U$ and $X$-variables in [GZ18] are respectively our $x$ - and $z$-variables), an analysis of the presentation of $A_{\Delta}$ exhibited in [GZ18, Theorem 3.2.5] reveals that $A_{\Delta}=R_{\Delta} /\left(y_{i}-x_{i} \mid i \in\{1, \ldots, n\}\right)$. Our first observation is then that $y_{1}-x_{1}, \ldots, y_{n}-x_{n}$ is a regular sequence of linear forms:

Proposition 8.1. Let $\Delta$ be a pure simplicial complex and let char $(\mathbb{F})=0$. Then $A_{\Delta}=$ $R_{\Delta} /\left(y_{i}-x_{i} \mid i \in\{1, \ldots, n\}\right)$, and $y_{1}-x_{1}, \ldots, y_{n}-x_{n}$ is an $R_{\Delta}$-regular sequence of linear forms.

Proof: Since $R_{\Delta}$ is Cohen-Macaulay, it is enough to prove that $\operatorname{dim} R_{\Delta}=n+\operatorname{dim} R_{\Delta} /\left(y_{i}-x_{i} \mid\right.$ $i \in\{1, \ldots, n\})$, see e.g. the graded version of [BH98, Theorem 2.1.2.c]. Since $R_{\Delta} /\left(y_{i}-x_{i} \mid\right.$ $i \in\{1, \ldots, n\})$ is Artinian and the Krull dimension of $R_{\Delta}$ equals $n$ (check Notation 4.1), the claim follows.

Corollary 8.2. Let $\Delta$ be a pure flag simplicial complex and let char $(\mathbb{F})=0$. Then the following conditions are equivalent:

i. $A_{\Delta}$ is Koszul;

ii. $R_{\Delta}$ is Koszul;

iii. $\Delta$ is Cohen-Macaulay over $\mathbb{F}$.

Proof: $\quad$ Conditions ii and iii are equivalent by Corollary 5.3. If $B$ is a standard graded $\mathbb{F}$-algebra and $\ell \in B_{1}$ is a regular element, one has that $B$ is Koszul if and only if $B / \ell B$ is

\footnotetext{
${ }^{2}$ This is only needed in order to use Macaulay's inverse system, but does not really play a role in our observations: see Remark 8.4.
} 
[BF85, Theorem 4.e.iv]; hence, the equivalence of conditions i and ii descends directly from Proposition 8.1.

If $B$ is a standard graded $\mathbb{F}$-algebra, $\ell \in B_{1}$ is a regular element and $B / \ell B$ has a quadratic Gröbner basis, then so does $B$ [Con00, Lemma 4]; the reverse implication does not hold in general. In our case, however, the proof of the "only if" implication of Theorem 6.3 goes through for $A_{\Delta}$ as well: the only difference lies in the presence of some new nontrivial $S$-pairs, namely the ones coming from a nonface-monomial $\mathbf{x}^{N}$ and a binomial $\mathbf{x}^{F_{1} \backslash F_{2}} z_{F_{1}}-\mathbf{x}^{F_{2} \backslash F_{1}} z_{F_{2}}$. It can be checked that such $S$-pairs reduce to zero. Putting these observations together, we get the following result:

Proposition 8.3. Let $\Delta$ be a pure flag simplicial complex and let char $(\mathbb{F})=0$. Then the following conditions are equivalent:

i. $A_{\Delta}$ has a quadratic Gröbner basis;

ii. $R_{\Delta}$ has a quadratic Gröbner basis;

iii. $\Delta$ is shellable.

Remark 8.4. The running hypothesis of this section about characteristic zero is needed just to fit the original definition of $A_{\Delta}$ by Gondim and Zappalà, where Macaulay's inverse system is used. However, the proof that $y_{1}-x_{1}, \ldots, y_{n}-x_{n}$ is an $R_{\Delta}$-regular sequence of linear forms is characteristic-free, and so are the proofs of Corollary 8.2 and Proposition 8.3, if we substitute " $A_{\Delta}$ " by " $R_{\Delta} /\left(y_{i}-x_{i} \mid i \in\{1, \ldots, n\}\right)$ " in the statements. In particular, the results in Section 5.3 and Section 7 can be adapted to fit the Artinian setting.

Remark 8.5. Because of Proposition 8.1, the algebra $A_{\Delta}$ has a quadratic defining ideal precisely when $R_{\Delta}$ does; in particular, the characterizations of quadraticity in [GZ18, Theorem 3.5] and in our Proposition 4.4 should coincide. However, Proposition 4.4 requires $\Delta$ to be flag and $\left(S_{2}\right)$, while [GZ18, Theorem 3.5] only asks for $\Delta$ to be flag and strongly connected, which is weaker.

We claim that the complex $\Delta$ with facet list $\{123,235,245,457,567,167\}$ provides a counterexample to [GZ18, Theorem 3.5]: indeed, $\Delta$ is strongly connected but not $\left(S_{2}\right)$, and the cubical generator $x_{2} x_{3} z_{123}-x_{6} x_{7} z_{167}$ is needed in the presentation of $A_{\Delta}$, as can be checked for instance with Macaulay2 using the InverseSystems package [EB].

Note that the examples constructed via Turán complexes in [GZ18] are still valid: since Turán complexes are flag and Cohen-Macaulay, by Corollary 8.2 the associated algebras are Koszul, and hence quadratically presented.

\section{FUNDING}

The first-named author was partially supported by the EPSRC grant EP/R02300X/1. The second-named author is funded by the Göran Gustafsson foundation.

\section{ACKNOWLEDGEMENTS}

The first seeds for this paper were planted in June 2019, when the first-named author found counterexamples to the algebraic generalization of the Charney-Davis conjecture mentioned above via some Macaulay2 [GS21] experiments. The project then took shape in December 
2019, while the first-named author was visiting the second-named author at the Max Planck Institute for Mathematics in the Sciences in Leipzig, Germany. The first-named author is grateful to the institute for the hospitality.

We wish to thank Aldo Conca, Rodrigo Gondim, Vic Reiner, Naoki Terai, Matteo Varbaro, Volkmar Welker and Giuseppe Zappalà for useful discussions, and an anonymous referee for their thoughtful comments. Special thanks go to Eran Nevo for an insightful exchange of emails which put us on the right track. Finally, we thank Bruno Vallette for pointing us to further connections between the Koszul and Cohen-Macaulay properties in the literature.

\section{REFERENCES}

[Ath18] Christos A. Athanasiadis. Gamma-positivity in combinatorics and geometry. Sém. Lothar. Combin., 77:Art. B77i, 64, [2016-2018].

[BF85] Jörgen Backelin and Ralf Fröberg. Koszul algebras, Veronese subrings and rings with linear resolutions. Rev. Roumaine Math. Pures Appl., 30(2):85-97, 1985.

[BH98] Winfried Bruns and Jürgen Herzog. Cohen-Macaulay rings, volume 39 of Cambridge Studies in Advanced Mathematics. Cambridge University Press, Cambridge, 1998. Second edition.

[Bie92] Thomas Bier. A remark on Alexander duality and the disjunct join. 1992. Preprint.

[Bjö95] Anders Björner. Topological methods. In Handbook of combinatorics, volume 1,2, pages 18191872. 1995.

$\left[\mathrm{BOW}^{+} 20\right]$ Christin Bibby, Andrew Odesky, Mengmeng Wang, Shuyang Wang, Ziyi Zhang, and Hailun Zheng. Minimal flag triangulations of lower-dimensional manifolds. Involve, 13(4):683-703, 2020.

[BPSZ05] Anders Björner, Andreas Paffenholz, Jonas Sjöstrand, and Günter M. Ziegler. Bier spheres and posets. Discrete Comput. Geom., 34(1):71-86, 2005.

[Brä06] Petter Brändén. Sign-graded posets, unimodality of $W$-polynomials and the Charney-Davis conjecture. Electron. J. Combin., 11(2):Research Paper 9, 15, 2004/06.

[CD95] Ruth Charney and Michael Davis. The Euler characteristic of a nonpositively curved, piecewise Euclidean manifold. Pacific J. Math., 171(1):117-137, 1995.

[CDNR13] Aldo Conca, Emanuela De Negri, and Maria Evelina Rossi. Koszul algebras and regularity. In Commutative algebra, pages 285-315. Springer, New York, 2013.

[Con00] Aldo Conca. Gröbner bases for spaces of quadrics of low codimension. Adv. in Appl. Math., 24(2):111-124, 2000.

[CV13] Alexandru Constantinescu and Matteo Varbaro. On the $h$-vectors of Cohen-Macaulay flag complexes. Math. Scand., 112(1):86-111, 2013.

[D'A17] Alessio D'Alì. The Koszul property for spaces of quadrics of codimension three. J. Algebra, 490:256-282, 2017.

[DFN19] Alessio D'Alì, Gunnar Fløystad, and Amin Nematbakhsh. Resolutions of co-letterplace ideals and generalizations of Bier spheres. Trans. Amer. Math. Soc., 371(12):8733-8753, 2019.

[DMV19] Hailong Dao, Linquan Ma, and Matteo Varbaro. Regularity, singularities and $h$-vector of graded algebras. arXiv preprint arXiv:1901.01116, 2019.

[EB] David Eisenbud and Mats Boij. InverseSystems: equivariant Macaulay inverse systems. Version 1.1. A Macaulay2 package available at https://github.com/Macaulay2/M2/tree/master/ M2/Macaulay2/packages.

[Eis95] David Eisenbud. Commutative Algebra: With a View Toward Algebraic Geometry, volume 150 of Graduate Texts in Mathematics. Springer-Verlag, New York, 1995.

[ER98] John A. Eagon and Victor Reiner. Resolutions of Stanley-Reisner rings and Alexander duality. J. Pure Appl. Algebra, 130(3):265-275, 1998.

[For07] Robin Forman. Topics in combinatorial differential topology and geometry. In Geometric combinatorics, volume 13 of IAS/Park City Math. Ser., pages 133-205. Amer. Math. Soc., Providence, RI, 2007.

[Frö75] Ralph Fröberg. Determination of a class of Poincaré series. Math. Scand., 37(1):29-39, 1975. 
[Gal05] Światosław R. Gal. Real root conjecture fails for five- and higher-dimensional spheres. Discrete Comput. Geom., 34(2):269-284, 2005.

[Gou99] Henry W. Gould. The Girard-Waring power sum formulas for symmetric functions and Fibonacci sequences. Fibonacci Quart., 37(2):135-140, 1999.

[GP08] Gert-Martin Greuel and Gerhard Pfister. A Singular introduction to commutative algebra. Springer-Verlag, Berlin, 2008. With contributions by Olaf Bachmann, Christoph Lossen and Hans Schönemann. Second, extended edition.

[GS21] Daniel R. Grayson and Michael E. Stillman. Macaulay2, a software system for research in algebraic geometry. Available at http://www.math.uiuc.edu/Macaulay2/, 2021.

[Gul72] Tor H. Gulliksen. Massey operations and the Poincaré series of certain local rings. J. Algebra, 22:223-232, 1972.

[GZ18] Rodrigo Gondim and Giuseppe Zappalà. Lefschetz properties for Artinian Gorenstein algebras presented by quadrics. Proc. Amer. Math. Soc., 146(3):993-1003, 2018.

[Har62] Robin Hartshorne. Complete intersections and connectedness. Amer. J. Math., 84:497-508, 1962.

[Hat02] Allen Hatcher. Algebraic topology. Cambridge University Press, Cambridge, 2002.

[HK12] Inga Heudtlass and Lukas Katthän. Algebraic properties of Bier spheres. Matematiche (Catania), 67(1):91-101, 2012.

[IK99] Anthony Iarrobino and Vassil Kanev. Power sums, Gorenstein algebras, and determinantal loci, volume 1721 of Lecture Notes in Mathematics. Springer-Verlag, Berlin, 1999. Appendix C by Iarrobino and Steven L. Kleiman.

[Inc21] OEIS Foundation Inc. The On-Line Encyclopedia of Integer Sequences. http://oeis.org/, 2021.

[Mat18] Kazunori Matsuda. Non-Koszul quadratic Gorenstein toric rings. Math. Scand., 123(2):161-173, 2018.

[MP15] Jason McCullough and Irena Peeva. Infinite graded free resolutions. In Commutative algebra and noncommutative algebraic geometry. Vol. I, volume 67 of Math. Sci. Res. Inst. Publ., pages 215-257. Cambridge Univ. Press, New York, 2015.

[MS05] Ezra Miller and Bernd Sturmfels. Combinatorial commutative algebra, volume 227 of Graduate Texts in Mathematics. Springer-Verlag, New York, 2005.

[MS20] Jason McCullough and Alexandra Seceleanu. Quadratic Gorenstein algebras with many surprising properties. Arch. Math. (Basel), 115(5):509-521, 2020.

[MSS21a] Matthew Mastroeni, Hal Schenck, and Mike Stillman. Quadratic Gorenstein rings and the Koszul property I. Trans. Amer. Math. Soc., 374(2):1077-1093, 2021.

[MSS21b] Matthew Mastroeni, Hal Schenck, and Mike Stillman. Quadratic Gorenstein rings and the Koszul property II. Int. Math. Res. Not., 2021+. Published online on October 14th, 2021. https://doi . org/10.1093/imrn/rnab297.

[MT09] Satoshi Murai and Naoki Terai. $h$-vectors of simplicial complexes with Serre's conditions. Math. Res. Lett., 16(6):1015-1028, 2009.

[Mur11] Satoshi Murai. Spheres arising from multicomplexes. J. Combin. Theory Ser. A, 118(8):21672184, 2011.

[Pol95] Patrick Polo. On Cohen-Macaulay posets, Koszul algebras and certain modules associated to Schubert varieties. Bull. London Math. Soc., 27(5):425-434, 1995.

[PRS98] Irena Peeva, Victor Reiner, and Bernd Sturmfels. How to shell a monoid. Math. Ann., 310(2):379393, 1998.

[PS09] Irena Peeva and Mike Stillman. Open problems on syzygies and Hilbert functions. J. Commut. Algebra, 1(1):159-195, 2009.

[Rei76] Gerald Allen Reisner. Cohen-Macaulay quotients of polynomial rings. Advances in Math., 21(1):30-49, 1976.

[Roo93] Jan-Erik Roos. Commutative non-Koszul algebras having a linear resolution of arbitrarily high order. Applications to torsion in loop space homology. C. R. Acad. Sci. Paris Sér. I Math., 316(11):1123-1128, 1993. 
[RS10] Victor Reiner and Dumitru Ioan Stamate. Koszul incidence algebras, affine semigroups, and Stanley-Reisner ideals. Adv. Math., 224(6):2312-2345, 2010.

[RW05] Victor Reiner and Volkmar Welker. On the Charney-Davis and Neggers-Stanley conjectures. $J$. Combin. Theory Ser. A, 109(2):247-280, 2005.

[Sta77] Richard P. Stanley. Cohen-Macaulay complexes. In Higher combinatorics (Proc. NATO Advanced Study Inst., Berlin, 1976), pages 51-62. NATO Adv. Study Inst. Ser., Ser. C: Math. and Phys. Sci., 31, 1977.

[Sta96] Richard P. Stanley. Combinatorics and commutative algebra, volume 41 of Progress in Mathematics. Birkhäuser Boston, Inc., Boston, MA, second edition, 1996.

[Ter07] Naoki Terai. Alexander duality in Stanley-Reisner rings. In Affine algebraic geometry, pages 449-462. Osaka Univ. Press, Osaka, 2007.

[Val07] Bruno Vallette. Homology of generalized partition posets. J. Pure Appl. Algebra, 208(2):699-725, 2007.

[Vil90] Rafael H. Villarreal. Cohen-Macaulay graphs. Manuscripta Math., 66(3):277-293, 1990.

[Woo98] David Woodcock. Cohen-Macaulay complexes and Koszul rings. J. London Math. Soc. (2), 57(2):398-410, 1998.

[Yan00] Kohji Yanagawa. Alexander duality for Stanley-Reisner rings and squarefree $\mathbf{N}^{n}$-graded modules. J. Algebra, 225(2):630-645, 2000.

Institut für Mathematik, Universität Osnabrück, 49069 Osnabrück, Germany (CurRent aDDRESS) AND

Mathematics Institute, University of Warwick, CV4 7AL Coventry, United Kingdom

Email address: alessio.dali@uni-osnabrueck.de

KTH Royal Institute of Technology, 11428 Stockholm, Sweden

Email address: lven@kth.se lorenzo.venturello@hotmail.it 